\title{
MobC of conjugative RA3 plasmid from IncU group autoregulates the expression of bicistronic mobC-nic operon and stimulates conjugative transfer
}

\author{
Jolanta Godziszewska', Anna Kulińska ${ }^{1,2}$ and Grażyna Jagura-Burdzy ${ }^{1 *}$
}

\begin{abstract}
Background: The IncU conjugative transfer module represents highly efficient promiscuous system widespread among conjugative plasmids of different incompatibility groups. Despite its frequent occurrence the mechanisms of relaxosome formation/action are far from understood. Here we analyzed the putative transfer auxiliary protein MobC of the conjugative plasmid RA3 from the IncU incompatibility group.

Results: MobC is a protein of 176 amino acids encoded in the bicistronic operon mobC-nic adjacent to oriT. MobC is homologous to prokaryotic transcription factors of the ribbon-helix-helix $(\mathrm{RHH})$ superfamily. Conserved LxxugxNINQiaxxLn motif clusters MobC with the clade of conjugative transfer auxilliary proteins of Mobp relaxases. MobC forms dimers in solution and autoregulates the expression of mobCp by binding to an imperfect palindromic sequence $\left(\mathrm{O}_{M}\right)$ located between putative -35 and -10 motifs of the promoter. Medium-copy number test plasmid containing the oriT-mobCp region is mobilized with a high frequency by the RA3 conjugative system. The mutations introduced into $\mathrm{O}_{M}$ that abolished MobC binding in vitro decreased 2-3 fold the frequency of mobilization of the test plasmids. The deletion of $\mathrm{O}_{M}$ within the RA3 conjugative module had no effect on transfer if the mobC-nic operon was expressed from the heterologous promoter. If only nic was expressed from the heterologous promoter (no mobC) the conjugative transfer frequency of such plasmid was 1000-fold lower.
\end{abstract}

Conclusion: The MobC is an auxiliary transfer protein of dual function. It autoregulates the expression of mobC-nic operon while its presence significantly stimulates transfer efficiency.

Keywords: Broad-host range plasmid, Conjugative transfer, IncU, MobC, Transfer auxiliary protein

\section{Background}

Broad-host-range conjugative plasmids are considered the main factors responsible for the horizontal spreading of genetic information between distantly related bacterial species. Although the conjugation process has been fairly well described for some model systems like F, Ti, R388 or RK2 [1-5], its regulation and the environmental stimuli responsible for the initiation of conjugation remain elusive. In Gram-negative bacteria the conjugation functions comprise processing of DNA for transfer (Dtr)

\footnotetext{
* Correspondence: gjburdzy@ibb.waw.pl

'Institute of Biochemistry and Biophysics, Department of Microbial Biochemistry, PAS, 02-106 Warsaw, Poland

Full list of author information is available at the end of the article
}

and mating pair formation (Mpf). Among Dtr proteins the pivotal role is played by relaxase which recognizes a specific motif in oriT (origin of transfer), nicks a single DNA strand, covalently binds to the $5^{\prime}$ end of the transferred strand (T-DNA) and re-joins the ends after ssDNA translocation to the recipient. Proteins involved in Dtr and Mpf functions form two large complexes: relaxosome (relaxase bound at specific DNA sequence oriT and auxiliary proteins) [5] and membrane located transferosome (type IV secretion system, T4SS) [6]. The third essential element of conjugative transfer system is a coupling protein $(\mathrm{T} 4 \mathrm{CP})$ that links the relaxosome with the transferosome [7]. The auxiliary proteins help to determine the specificity of oriT recognition by relaxase, enhance its 
nicking activity, stimulate ATPase activity of the coupling protein and act as transcriptional regulators for conjugative transfer operons [8-19].

The broad-host-range conjugative plasmid RA3, the archetype of the IncU incompatibility group, has been isolated from the fish pathogen Aeromonas hydrophila as the determinant of its antibiotic resistance [20]. RA3 nucleotide sequence has been determined [GenBank: DQ401103]; [21] and shown to be almost identical in its backbone part to another IncU representative pFBAOT6 [22]. The RA3 plasmid has been shown to be capable of replicating and self-transmitting between $\alpha-, \beta$ - and $\gamma$ - proteobacteria with a very high efficiency [21]. The conjugative transfer functions of RA3 are clustered in a region of $23 \mathrm{~kb}$ organized in three transcriptional units (Figure 1A). The first operon encodes two DNA- transfer associated products (Dtr): a predicted DNA- binding protein MobC (176 amino acids) and the relaxase Nic (331 amino acids). The sequence corresponding to the putative nick site of oriT has been identified in silico upstream of the mobC-nic operon [21]. It was then confirmed that when an intergenic fragment of $417 \mathrm{bp}$ containing putative ori $T_{\mathrm{RA} 3}$ (Figure 1B) was cloned into
pUC18 [23], it facilitated plasmid mobilization by the RA3 with a high frequency [21].

The second transfer operon located on the opposite DNA strand encompasses 19 open reading frames encoding proteins mainly involved in the mating pair formation (Mpf), four putatively in Dtr functions (homologs of RP4 primases TraC3 and TraC4, TraD and DNA topoisomerase Top), and a homolog of the coupling protein VirD4 [21]. The third operon contains three orfs orf34, orf35 and orf36 (Figure 1A) encoding a predicted membrane associated proteins and a DNA binding protein [21], that fulfill the auxilliary transfer functions (J. Godziszewska, unpublished).

We initiated a functional analysis of the RA3 conjugative module to uncover the roles of individual elements in this extremely efficient transfer system [21] starting from the operon mobC-nic. The closest homologs of MobC from IncU plasmids [22,24] have been found encoded in the similarly organized transfer regions of promiscuous plasmids from a putative new plasmid group, designated PromA [25]. They all have been intuitively classified as the auxiliary transfer protein (Figure 2B) [25].

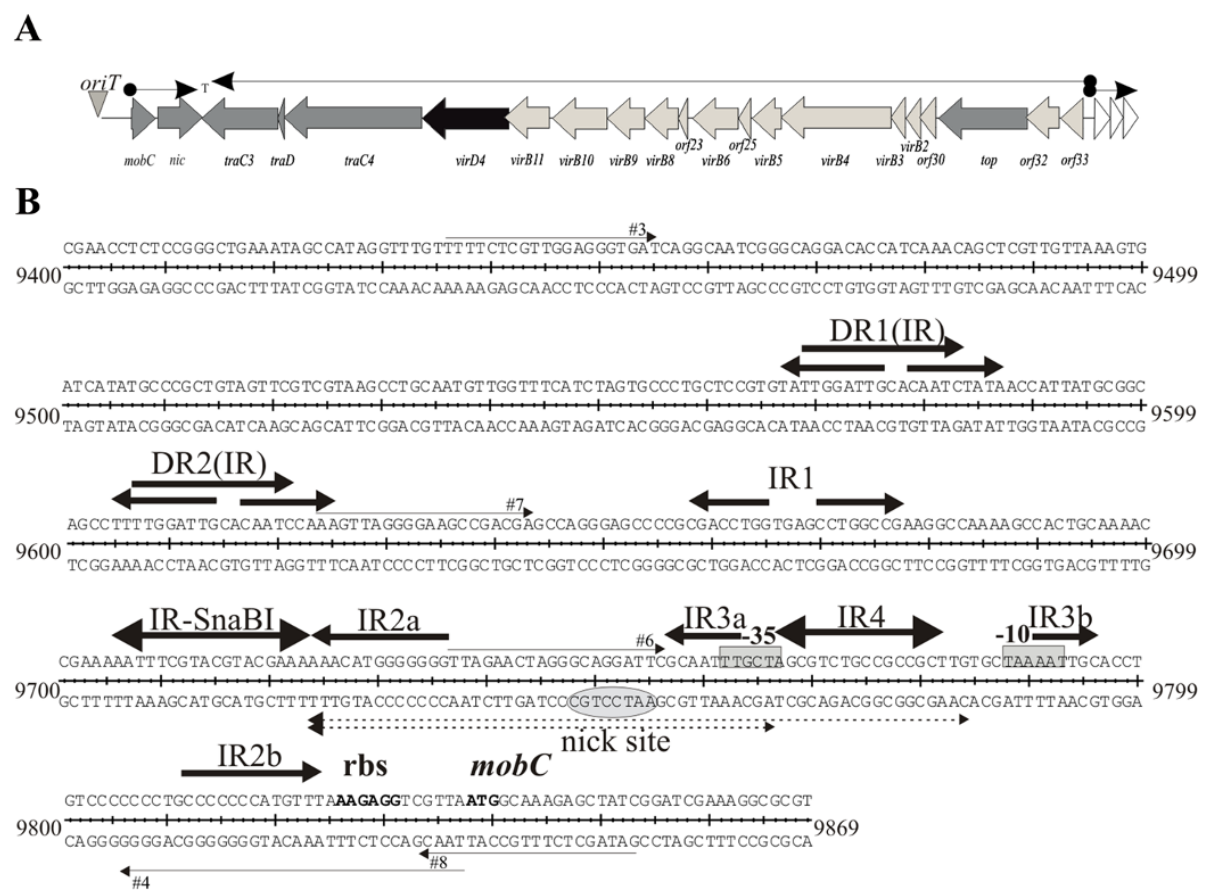

Figure 1 Organization of the RA3 conjugative transfer module. A. Transcriptional organization of the RA3 conjugative transfer module with orfs labeled according to the predicted function in the conjugation process: light grey arrows indicate homologs of Mpf system, dark grey arrows indicate proteins presumably involved in DNA replication and transfer (Dtr), and the black one marks a homolog of coupling protein VirD4. B. DNA sequence of mobCp/oriT/pars region between 9400 and 9869 nt of RA3 coordinates [GenBank: DQ401103]. Thin arrows correspond to primers used in the construction of deletion derivatives by PCR. Putative promoter motifs are boxed, and putative regulatory sequences are indicated by arrows. The inverted repeat IR-SnaBI is a part of the centromere-like sequence as the binding site for partitioning protein $\mathrm{Kor} B\left(\mathrm{O}_{\mathrm{B}}\right)$ [43]. Highly conserved nick site is circled and the ribosome binding site and initiation codon for MobC are in bold. The oligonucleotides tested as orits (61 nt and $45 \mathrm{nt}$ ) are shown. 
A

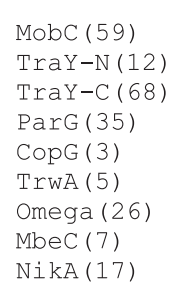

\section{B}

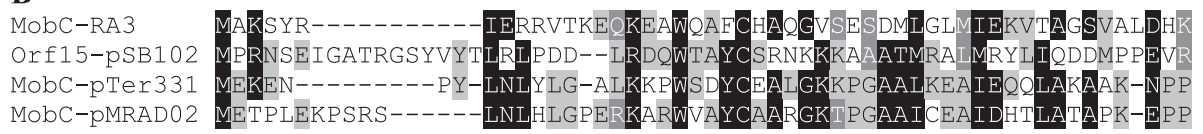
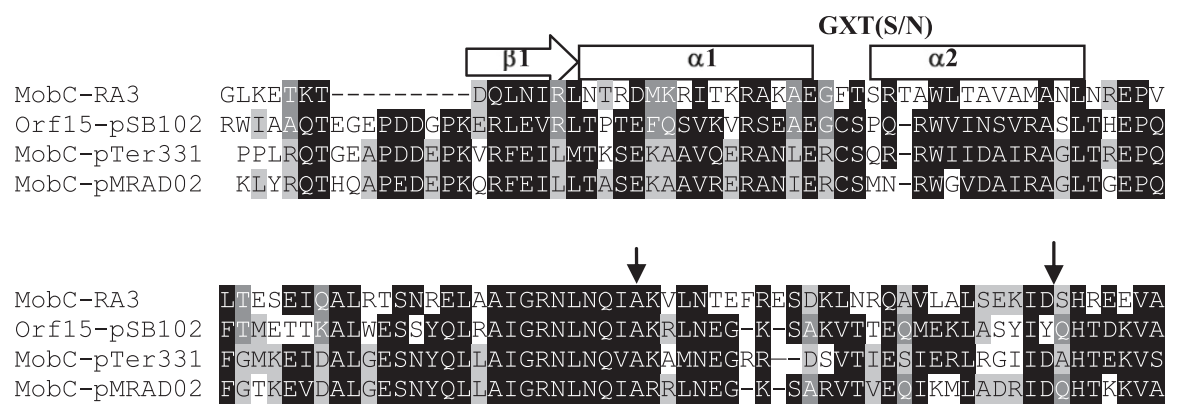

bacterial mobilization protein motif

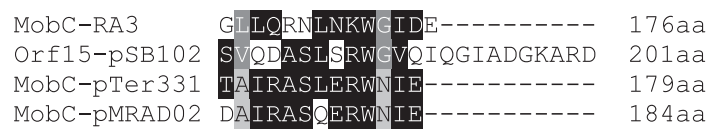

Figure 2 Amino acid sequence of MobC protein from RA3 (IncU). A. Ribbon-helix-helix (RHH) motifs of the plasmid proteins involved in conjugative transfer or stable maintenance: TraY of F [Uniprot:P06627]; ParG of TP228 [Uniprot: Q70W75], CopG of Streptococcus plasmid pLS1 [Uniprot:P13920], TrwA of R388 [Uniprot: Q04229], Omega of pSM19035 [Uniprot: Q83UM6], MbeC of ColE1 [Uniprot: P13657] and NikA of R64 [Uniprot: Q79W8]. The numbers in brackets correspond to the first amino acid residue of RHH motif. Similar residues in seven to nine representatives are shadowed in white on black, those similar in four to six representatives are shadowed grey. B. MobC family of putative conjugative auxiliary proteins from the IncU plasmids (due to the high similarity only MobC of RA3 is shown as the representative of IncU group) [GenBank: ABD64841] and PromA groups (Orf15 of pSB102 [GenBank: NP_361029], MobC of pTer331 [GenBank: YP_001672038] and MobC of pMRAD02 [GenBank: ACB28263]. Putative RHH motif and a highly conserved "mobilization protein motif" are shown. Similar residues in three or four representatives are shadowed in white on black, those similar in two are shadowed grey. Black arrows mark the C-termini of truncated MobCs analyzed in this work.

The best characterized auxiliary transfer proteins are TraJ, TraK and TraH of RK2 [26], TraY and TraM of F [8,10,27], TrwA of R388 [28] and MbeC of ColE1 [13]. They ensure specificity of the relaxase binding to the nick site, change the topology of DNA by bending, enhance the nicking reaction, stimulate unwinding of oriT, and stabilize the relaxosome [8-12]. It has been shown that C-terminal domain of TrwA stimulates ATPase activity of TrwB, the coupling protein of R388 conjugative system [14]. The role of the auxiliary proteins in the relaxosome formation/activity is essential since deletions of the coding regions or their binding sites decreased up to $10^{5}$ the frequency of plasmid mobilization $[19,29,30]$. In many cases the auxiliary transfer proteins also act as transcription factors repressing or activating the expression of tra genes [15-17,19].

In this work we have analyzed the role of MobC in the conjugative transfer of RA3 plasmid showing that it is an auxiliary transfer protein of dual function. MobC controls the expression of mobC-nic operon by binding to the operator sequence in the $m o b C p$ and is required for the fully efficient conjugation process.

\section{Methods}

Bacterial strains and growth conditions

Escherichia coli strains used were DH5 $\alpha\left[\mathrm{F}^{-}(\Phi 80 \mathrm{~d} l a c Z \Delta M 15)\right.$ recA1 endA1 gyrA96 thi-1 hsdR17(ri $\left.m_{k}^{+}\right)$supE44 relA1 deoR 
$\triangle\left(\right.$ lacZYA-argF)U196] and its Rif ${ }^{\mathrm{R}}$ derivative, BL21 [F $\mathrm{F}^{-}$ompT $h s d S_{\mathrm{B}}\left(\mathrm{r}_{\mathrm{B}}^{-} \mathrm{m}_{\mathrm{B}}^{-}\right)$gal dcm ( $\lambda$ DE3)] (Novagen, 2003); and BTH101 [F- cya-99 araD139 galE15 galK16 rpsL1 $\left(\mathrm{Sm}^{\mathrm{R}}\right)$ hsdR2 mcrA1 mcrB1] [31]. Bacteria were grown in L-broth [32] at $37^{\circ} \mathrm{C}$ or on L-agar (L-broth with $1.5 \% \mathrm{w} / \mathrm{v}$ agar) supplemented with appropriate antibiotics: benzyl penicillin, sodium salt $\left(150 \mu \mathrm{g} \mathrm{ml}^{-1}\right.$ in liquid media and $300 \mu \mathrm{g} \mathrm{ml}^{-1}$ in agar plates) for penicillin resistance, kanamycin $50 \mu \mathrm{g} \mathrm{ml}^{-1}$ for kanamycin resistance and chloramphenicol $10 \mu \mathrm{g} \mathrm{ml}^{-1}$ for chloramphenicol resistance. MacConkey Agar Base (Difco) supplemented with $1 \%$ maltose was used for bacterial adenylate cyclase two-hybrid (BACTH) system. L agar used for blue/white screening contained IPTG $(0.1 \mathrm{mM})$ and X-gal $\left(40 \mu \mathrm{g} \mathrm{ml}^{-1}\right)$.

Plasmid DNA isolation, analysis, cloning and manipulation Plasmid DNA was isolated and manipulated by standard procedures [33]. Plasmids used and constructed in this study are listed in Table 1 . Standard PCR reactions [34] were performed with pairs of primers listed in the Additional file 1. PCR reactions to amplify mobC were performed with an initial denaturation step $\left(95^{\circ} \mathrm{C}\right.$ for 5 minutes) and 25 cycles of denaturation at $95^{\circ} \mathrm{C}$ for 30 seconds, annealing at $55^{\circ} \mathrm{C}$ for 30 seconds and elongation at $72^{\circ} \mathrm{C}$ for 40 seconds; for amplification of mobCp fragment and its derivatives annealing was performed at $59^{\circ} \mathrm{C}$ for 30 seconds. Reactions ended with a final elongation step $\left(72^{\circ} \mathrm{C}\right.$ for 7 minutes). All PCR- derived clones were sequenced to verify the nucleotide sequence.

The high-copy number expression vector pGBT30 [35], based on the pMB1 replicon with $l a c I^{\mathrm{q}}$ and tacp was used for regulated expression of mobC derivatives. The tacp-mobC transcriptional fusion with $l a c I^{\mathrm{q}}$ was recloned also into the medium copy number broad-host range vector pBBR1MCS-1 [36].

The mutant mobC1-129 allele resulted from a spontaneous nucleotide substitution introducing a stop codon after A129 of MobC. The deletion mutant mobC1-155 was constructed by cleavage of pJSB8.1 by ClaI, filling-in the $5^{\prime}$ overhangs and re-ligation. This led to the $\mathrm{N}$ terminal 155 amino acids from MobC being extended by three residues. Plasmids for over-expression and purification of the MobC derivatives were constructed by inserting the $m o b C$ variants as EcoRI-SalI fragments into pET28a $\mathrm{Km}^{\mathrm{R}}$ (Novagen).

\section{Site-directed mutagenesis in vitro}

To introduce mutations in the oriT-mobCp region an in vitro PCR- based site-directed mutagenesis method (Stratagene) was used with the high fidelity PfuTurbo DNA polymerase. Pairs of complementary primers \#9/ $\# 10$, \#11/\#12, \#13/\#14 and \#15/\#16 (Additional file 1) were designed to introduce nucleotide substitutions in a particular region of the amplified plasmid DNA accompanied by either removal of an existing restriction site or introduction of a new one to facilitate screening. Candidate mutant plasmids were tested for the presence/absence of the restriction site affected by the mutagenic primers and the correctness of mutagenesis was verified by sequencing.

\section{Bacterial transformation}

Competent cells of $E$. coli were prepared by standard $\mathrm{CaCl}_{2}$ method [33].

Determination of catechol 2,3-dioxygenase activity (XyIE) XylE activity (the product of $x y l E$ ) was assayed in extracts from logarithmically growing cultures. The overnight cultures were used to inoculate $25 \mathrm{ml}$ of L-broth (dilution 1:50) supplemented with antibiotics and $0.5 \mathrm{mM}$ IPTG when needed. Cultures were grown for $1.5 \mathrm{~h}$ to $3 \mathrm{hrs}$ at $37^{\circ} \mathrm{C}$, centrifuged and pellets were resuspended in $500 \mu \mathrm{l}$ of $0.1 \mathrm{M} \mathrm{KPi}$ buffer (pH 7.5) and $50 \mu \mathrm{l}$ of acetone and left on ice. After sonication the extracts were cleared by centrufugationat $16000 \times \mathrm{g}$ for $15 \mathrm{~min}$ at $4^{\circ} \mathrm{C}$. XylE activity was assayed spectrophotometrically according to Zukowski method [37]. The reaction was initiated by addition of $0.2 \mathrm{mM}$ catechol solution. One unit of catechol 2,3- dioxygenase activity is defined as the amount of enzyme needed to convert $1 \mu \mathrm{mol}$ of catechol to 2-hydroxymuconic semialdehyde in 1 minute per $\mathrm{mg}$ of protein. Protein concentration was determined using the Bradford method [38].

\section{Purification of $\mathrm{His}_{6}$-tagged MobC derivatives}

For protein over-production and purification, E. coli BL21(DE3) was transformed with pET28 derivatives encoding $\mathrm{N}$-terminally $\mathrm{His}_{6}$-tagged MobCs. The overnight inoculum of the transformant was diluted 1:50 to $500 \mathrm{ml}$ of L-broth with kanamycin and cultured with shaking at $37^{\circ} \mathrm{C}$ for 1.5 hours. Then the $0.5 \mathrm{mM}$ IPTG was added and culture left to grow for two hours. The cells were pelleted by centrifugation, suspended in $1 \mathrm{ml}$ of sonication buffer ( $50 \mathrm{mM}$ sodium phosphate $\mathrm{pH} 8.0,300 \mathrm{mM} \mathrm{NaCl}$ ), and disrupted by sonication. The extract was purified by affinity chromatography as described previously [39] with the use of Protino column (Macherey-Nagel). Eluted protein fractions were analyzed by SDS-PAGE using a PHAST system (Pharmacia) with $20 \%$ homogeneous gels.

\section{Analysis of protein-DNA interactions by electrophoretic mobility shift assay (EMSA)}

PCR- amplified DNA fragments (417 bp) of modified variants of the oriT-mobCp region were excised from agarose gels and purified using the Gel-Out kit (A\&A Biotechnology). The protein-DNA binding reactions were performed for $15 \mathrm{~min}$ at $37^{\circ} \mathrm{C}$ in binding buffer 
Table 1 Plasmids used in this study

\begin{tabular}{|c|c|c|c|}
\hline Designation & Relevant features & Copy number & References \\
\hline PBBR1MCS-1 & $\mathrm{BHR}^{1}, \operatorname{InCA} / \mathrm{C}, \mathrm{Cm}^{\mathrm{R}}$ & Medium & {$[36]$} \\
\hline pBGS18 & ori $_{M B 1}, \mathrm{Km}^{\mathrm{R}}$ & High & [41] \\
\hline pET28a & ori $_{\mathrm{MB} 1}, \mathrm{Km}^{\mathrm{R}}, \mathrm{T} 7 \mathrm{p}, \mathrm{lacO}, \mathrm{His}_{6}-\mathrm{tag}, \mathrm{T} 7 \mathrm{tag}$ & Medium & Novagen \\
\hline pGBT30 & ori $_{M B 1}, A p^{R}$, lacl $\left.\right|^{q}$ tacp expression vector & High & [35] \\
\hline pJSB1.24 & ori $_{\mathrm{MB} 1}, \mathrm{Km}^{\mathrm{R}}$, Tra $\mathrm{RA}_{3}$-korCp-korC (RA3 coordinates $\left.9437-33657 \mathrm{nt}, 3093-3705 \mathrm{nt}\right)$ & High & {$[42]$} \\
\hline pKGB4 & pUT18 with MCS modified & High & Głąbski K. $^{2}$ \\
\hline pKGB5 & pKNT25 with MCS modified & Medium & Głąbski K.2 $^{2}$ \\
\hline pKNT25 & ori $_{p 15}, \mathrm{Km}^{\mathrm{R}}$, lacp -MCS - cyaT25 & Medium & [31] \\
\hline pKT25 & ori $_{\mathrm{p} 15}, \mathrm{Km}^{\mathrm{R}}$, lacp-cyaT25-MCS & Medium & {$[31]$} \\
\hline pKT25-zip & ori $_{p 15}, \mathrm{Km}^{\mathrm{R}}$, lacp-cyaT25-GCN4 leucine zipper & Medium & [31] \\
\hline pLKB2 & pKT25 with MCS modified & Medium & Kusiak L. ${ }^{2}$ \\
\hline$\overline{\mathrm{pLKB} 4}$ & pUT18C with MCS modified & High & Kusiak L. ${ }^{2}$ \\
\hline pMPB13.3 & ori $_{R A 3}, \mathrm{Km}^{R}$ pABB20-lac ${ }^{19}$-tacp-nic & Low & Przyłuski M. $^{2}$ \\
\hline pMPB13.4 & ori $_{\text {RA3, }}, \mathrm{Km}^{\mathrm{R}}$ pABB20-lacla-tacp-mobc-nic & Low & Przyłuski M. ${ }^{2}$ \\
\hline рPT01 & ori $_{s C 101}, \mathrm{Km}^{\mathrm{R}}$, promotorless $x y l E$ & Medium & {$[50]$} \\
\hline$\overline{\mathrm{RA3}}$ & $\mathrm{BHR}^{1}, \mathrm{InCU}, \mathrm{Cm}^{\mathrm{R}} \mathrm{Sm}^{\mathrm{R}}, \mathrm{Su}^{\mathrm{R}}$ & Low & Hayes F. ${ }^{3}$ \\
\hline pUC18 & ori $_{M B 1}, A p^{R}$ & High & [23] \\
\hline pUT18 & ori $_{\text {Cole1, }}$ Ap $^{R}$, lacp-MCS-cyaT18 & High & [31] \\
\hline pUT18C & ori $_{\text {ColE1, }} \mathrm{Ap}^{\mathrm{R}}$, lacp -cyaT18-MCS & High & [31] \\
\hline pUT18C-zip & ori $_{\text {ColE1, }} A p^{R}$, lacp -cyaT18-GCN4 leucine zipper & High & [31] \\
\hline
\end{tabular}

\section{Plasmids constructed in this study}

\section{Designation Description}

pJSB2.1 pUC18-mobC, EcoRl-Sall fragment amplified by PCR with the use of primers \#1 and \#2 (RA3 coordinates 9837-10455 nt)

pJSB2.2 pUC18-mobC1-129, EcoRI-Sall fragment amplified by PCR with the use of primers \#1 and \#2 spontaneous stop codon mutation at position 10225 nt (RA3 coordinates 9837-10455 nt)

pJSB2.9 pUC18-mobCp, 417 bp Sphl-BamHI fragment amplified by PCR with the use of primers \#3 and \#8 (RA3 coordinates 9435 -9852 nt)

pJSB2.11 pUC18 with 116 bp Sphl-BamHI PCR fragment amplified by PCR with the use of primers \#6 and \#8 (RA3 coordinates $9736-9852$ nt)

pJSB2.12 pUC18 with 116 bp Sphl-BamHI PCR fragment amplified by PCR with the use of primers \#5 and \#8 (RA3 coordinates $9736-9852 \mathrm{nt}$ ), mutation in a putative oriT motif

pJSB2.13 pUC18 with 100 bp Sphl-BamHI PCR fragment amplified with the use of primers \#5 and \#4 (RA3 coordinates 9736-9836 nt), mutation in a putative oriT motif

pJSB2.14 pUC18 with 100 bp Sphl-BamHI PCR fragment amplified with the use of primers \#6 and \#4 (RA3 coordinates $9736-9836 \mathrm{nt}$ )

pJSB2.15 PCR mutagenesis of pJSB2.9 with the use of primers \#9 and \#10 (mutVl)

pJSB2.16 PCR mutagenesis of pJSB2.9 with the use of primers \#13 and \#14 (mutVII)

pJSB2.17 PCR mutagenesis of pJSB2.9 with the use of primers \#11 and \#12 (mutVIII)

pJSB2.18 PCR mutagenesis of pJSB2.9 with the use of primers \#15 and \#16 (mut|X)

pJSB2.30 pUC18 mobC1-155, EcoRl-Sall fragment amplified by PCR with the use of primers \#1 and \#18 carrying (RA3 coordinates 9837-10308 nt)

pJSB2.43 pUC18 with 61 bp oligonucleotides (primer \#19 and \#20), oligonucleotides are inserted in Smal site in of pUC18

pJSB2.44 pJSB2.43 digested by Nhel and EcoRI, blunt-ended with the use of Klenow fragment and self-ligated, to remove IRIV

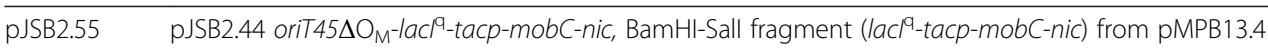

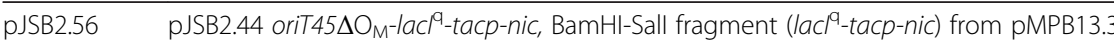

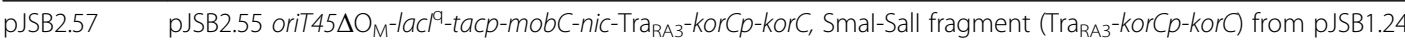

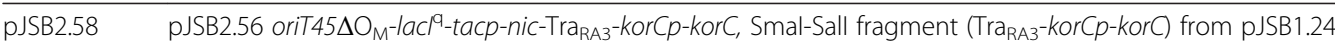

pJSB4.1 pBBR1MCS-1 lacl ${ }^{q}$, tacp-mobC, BamHI-Sall from pJSB5.1

pJSB4.2 pBBR1MCS-1 lacl' ${ }^{q}$, tacp-mobC1-129, BamHI-Sall from pJSB5.2

pJSB5.1 pGBT30 tacp-mobC, EcoRl-Sall fragment from pJSB6.1

pJSB5.2 pGBT30 tacp-mobC1-129, EcoRl-Sall fragment from pJSB6.2

pJSB6.1 pET28a T7p-mobC, EcoRI-Sall fragment from pJSB2.1 
Table 1 Plasmids used in this study (Continued)

\begin{tabular}{|c|c|}
\hline pJSB6.2 & pET28a T7p-mobC1-129, EcoRl-Sall fragment from pJSB2.2, \\
\hline pJSB6.30 & pET28a T7p-mobC1-155, EcoRI-Sall fragment from pJSB2.30 \\
\hline pJSB7.9 & pPTOI mobCp-xylE, Sphl-BamHI fragment from pJSB2.9 \\
\hline pJSB7.10 & pPT0I mobCp-xylE, 229 bp Sphl-BamHI fragment PCR amplified with the use of primers \#7 and \#8 (RA3 coordinates $9623-9852 \mathrm{nt})$ \\
\hline pJSB7.11 & pPTOI mobCp-xylE, Sphl-BamHI fragment from pJSB2.11 \\
\hline pJSB7.12 & pPTOI mobCp-xylE, Sphl-BamHI fragment from pJSB2.12 \\
\hline pJSB7.13 & pPTOI mobCp-xyIE, Sphl-BamHI fragment from pJSB2.13 \\
\hline pJSB7.14 & pPTOI mobCp-xyIE, Sphl-BamHI fragment from pJSB2.14 \\
\hline pJSB7.15 & pPTOI mobCp-xyIE, Sphl-BamHI fragment from pJSB2.15 \\
\hline pJSB7.16 & pPTOI mobCp-xyIE, Sphl-BamHI fragment from pJSB2.16 \\
\hline pJSB7.17 & pPTOI mobCp-xyIE, Sphl-BamHI fragment from pJSB2.17 \\
\hline pJSB7.18 & pPTOI mobCp-xyIE, Sphl-BamHI fragment from pJSB2.18 \\
\hline pJSB8.1 & pLKB4 cyaT18-mobC; EcoRI-Hincll fragment from pJSB6.1 cloned between EcoRI-Smal sites of pLKB4 \\
\hline pJSB8.30 & pJSB8.1 digested by Clal and self-ligated to remove 3' end of mobC (mobC1-155) \\
\hline pJSB9.1 & pKGB4 mobC-cyaT18, EcoRI-Sacl fragment amplified by PCR with primers \#1 and \#17 \\
\hline pJSB10.1 & pLKB2 cyaT25-mobC; EcoRl-Hincll fragment from pJSB5.1 cloned between EcoRI-Smal sites of pLKB2 \\
\hline pJSB10.2 & pLKB2 cyaT25-mobC1-129; EcoRI-Hincll fragment from pJSB5.2 cloned between EcoRI-Smal sites of pLKB2 \\
\hline pJSB11.1 & pKGB5 mobC-cyaT25; EcoRI-Sacl fragment from pJSB9.1.1 \\
\hline
\end{tabular}

$25 \mathrm{mM}$ Tris- $\mathrm{HCl} \mathrm{pH}$ 8.0; $10 \mathrm{mM} \mathrm{MgCl}_{2} ; 50 \mathrm{mM} \mathrm{NaCl}$; $0.1 \mathrm{mg} \mathrm{ml}^{-1} \mathrm{BSA}$ ) in a final volume of $20 \mu \mathrm{l}$ with increasing amounts of $\mathrm{His}_{6}$-MobC added. The MobC binding was analyzed on $1.2 \%$ agarose gels run in $1 x T B E$ buffer. The gels were stained with ethidium bromide and DNA visualized under UV light.

\section{Cross-linking with glutaraldehyde}

$\mathrm{His}_{6}$-tagged MobCs purified on $\mathrm{Ni}^{2+}$-agarose column were cross-linked with glutaraldehyde [39] and separated on $20 \%(\mathrm{w} / \mathrm{v})$ SDS-PAGE gel. The proteins were transferred onto a nitrocellulose membrane and Western blotting with anti-His tag antibodies was performed as described previously [40].

\section{Conjugation/ mobilization procedure}

In the mobilization experiments the donor strain $\mathrm{DH} 5 \alpha$ carried the RA3 or pJSB1.24 (pBGS18 [41] derivative with $\mathrm{Tra}_{\mathrm{RA} 3}$ module and $\operatorname{kor} C$ gene; [42] as the helper plasmid and the mobilizable pPT01 derivatives with the variants of oriT-mobCp region inserted. In conjugation experiments strains: DH $5 \alpha(\mathrm{pUC18}), \mathrm{DH} 5 \alpha(\mathrm{pJSB} 2.57)$, DH5 $\alpha$ (pJSB2.58)(pBBR1MCS-1), DH5 $\alpha$ (pJSB2.58)(pJSB4.1), DH5 $\alpha$ (pJSB2.58)(pJSB4.2) were used as donors.

$\mathrm{DH} 5 \alpha \mathrm{Rif}^{\mathrm{R}}$ strain was used as the recipient. Aliquots of $100 \mu \mathrm{l}$ of overnight cultures of the donor and recipient strains were mixed $(1: 1)$ and incubated on L-agar plates for 2 hours at $37^{\circ} \mathrm{C}$. Cells were scrapped, re-suspended in L-broth and $10 \mu \mathrm{l}$ aliquots of serial 10 -fold dilutions were spotted onto L-agar plates with $100 \mu \mathrm{g} \mathrm{ml}^{-1}$ rifampicin and $50 \mu \mathrm{g} \mathrm{ml}{ }^{-1}$ kanamycin to estimate the number of transconjugants. In parallel, $100 \mu \mathrm{l}$ of the donor strain overnight culture was incubated on L-agar plate for 2 hours at $37^{\circ} \mathrm{C}$, cells were scrapped, diluted and plated on L-agar or L-agar with antibiotics selective for the donor strain. The transfer frequency was calculated as the number of transconjugants per donor cell.

\section{Bacterial adenylate cyclase two-hybrid system (BACTH system)}

The dimerization of MobC in vivo was analyzed using the bacterial adenylate cyclase two-hybrid (BACTH) system in E. coli [31]. The MobC protein was fused translationally to CyaT18 fragment and to CyaT25 fragment at the $\mathrm{N}$ - or Cterminus using two pairs of compatible vectors (pUT18/ pKNT25 and pUT18C/ pKT25). E. coli BTH101, an adenylate cyclase deficient strain (cya), was co-transformed with the appropriate pairs of BACTH plasmids and plated on MacConkey medium suplemented with $1 \%(\mathrm{w} / \mathrm{v}) \mathrm{mal}-$ tose, $0.5 \mathrm{mM}$ IPTG and selective antibiotics. The plates were incubated for $48 \mathrm{~h}$ at $27^{\circ} \mathrm{C}$. The ability to ferment maltose indicated the CyaA reconstitution through interactions between the fused polypeptides.

\section{Results}

MobC is an autorepressor of the mobC-nic operon

The transfer module of RA3 (coordinates 9400-32300 nt) is located between the stability module and class I 
integron [21]. The first conjugative transfer operon, bicistronic mobC-nic, preceded directly by oriT, encodes MobC and relaxase Nic (Figure 1A).

The homologs of MobC from IncU plasmids (Figure 2B) have been found encoded in the promiscuous plasmids from PromA group [25], by loci positioned in the junction region between maintenance and conjugative transfer operons that display similar genetic organization as IncU plasmids [43]. The presence of the highly conserved structural motif LxxugxNINQiaxxLn in the C- terminal part classifies MobC and its PromA homologs [44-46], as the putative conjugative transfer auxiliary proteins of $\mathrm{MOB}_{\mathrm{P}}$ relaxases [13].

$\mathrm{MobC}_{\mathrm{RA} 3}$ is a putative DNA binding protein of 176 amino acids with predicted ribbon-helix-helix (RHH) motif (Figure 2A) according to the primary sequence analysis [47] and secondary structure modelling (I-TASSER, Additional file 2). A characteristic pattern of alternating hydrophilic-hydrophobic side chains is present along $\mathrm{N}$-terminal $\beta$-strand with hydrophobic side chains at conserved positions 3, 5, and 7 (Figure 2A) while a positively charged residue usually occurs at position 2 or 6 (in MobC Arginine is present at position 6). A second feature of the RHH motif is a conserved G-X-S/T/N sequence in the loop between helix $\alpha 1$ and helix $\alpha 2$ (GFT in $\mathrm{MobC}$ ). At least four hydrophobic residues are usually present in helix $\alpha 1$ and helix $\alpha 2$ that together with hydrophobic side chains at positions 3,5 , and 7 comprise the hydrophobic core of RHH motif [47]. In the MobC there are six hydrophobic residues in the predicted helices.

The RHH superfamily of prokaryotic DNA binding factors [47], encompasses several transfer auxiliary proteins like TrwA of plasmid R388 $\left(\mathrm{MOB}_{\mathrm{F}}\right)$, TraY of plasmid $\mathrm{F}\left(\mathrm{MOB}_{\mathrm{F}}\right)$, MbeC of ColEI $\left(\mathrm{MOB}_{\mathrm{HEN}}\right)$ and NikA of R64 $\left(\mathrm{MOB}_{\mathrm{P}}\right)[13,28,48,49]$.

To analyze the role of MobC in regulation of $m o b C p$ expression the 417-bp DNA fragment upstream of the $m o b C_{\mathrm{RA} 3}$ gene (Figure 1B) was cloned into a promoterprobe vector pPT01 [50] in front of the promoterless $x y l E$ cassette to construct pJSB7.9. Measurements of catechol 2,3-dioxygenase activity (XylE) in the extracts of DH5 $\alpha(\mathrm{pJSB} 7.9)$ cells from the exponential phase of growth demonstrated a moderate level of $m o b C p$ transcriptional activity ( $0.5 \mathrm{U}$ of XylE).

The $m o b C$ orf was cloned into the high-copy number expression vector pGBT30 [35] under the control of tacp (synthetic IPTG- inducible promoter), to construct pJSB5.1. Introduction of pJSB5.1 into the DH5 $\alpha$ (pJSB7.9 $m o b C p-x y l E)$ strain led to 20-fold repression of $m o b C p$ even without MobC over-production indicating that $\mathrm{MobC}$ is a very potent repressor. Induction of MobC synthesis in DH5 $\alpha$ (pJSB7.9)(pJSB5.1) by culturing in the presence of $0.5 \mathrm{mM}$ IPTG for two hours switched off mobCp completely $(<0.002 \mathrm{U})$. No decrease in XylE activity was observed when strain DH5 $\alpha$ (pJSB7.9) (pGBT30) was grown in the presence of IPTG.

\section{Mapping of MobC operator}

The $m o b C p$ sequences have been postulated previously as the TTGCTA and TAAAAT hexamers separated by 20 nt [43], motifs close to the consensus -35 and -10 sequences recognized by RNAP $^{70}$ (Figure 1B). The sequence corresponding to the putative nick site of oriT [21] maps $50 \mathrm{nt}$ upstream of the predicted transcription start point (tsp) for mobC. The analyzed region of 417 bp contains numerous structural motifs: two direct repeats DR1(IR) and DR2(IR) overlapped by inverted repeats and five additional inverted repeats designated IRSnaBI and IR1 to IR4 (Figure 1B). The IR-SnaBI motif was identified previously as the KorB-binding site in the parS region [43]. Two of the palindromic sequences, IR2 and IR3, have their arms separated by $75 \mathrm{nt}$ and $26 \mathrm{nt}$, respectively. To map $\mathrm{O}_{M}$ in the $m o b C p$ region a series of deletion derivatives was constructed by PCR amplification and the truncated fragments with intact putative promoter sequences were cloned into the promoterprobe vector pPT01 (Figure 3A). All these derivatives were analyzed for promoter activity in the presence of empty expression vector (pGBT30) and MobC produced in trans (pJSB5.1 tacp-mobC).

Some variability in the level of $m o b C p$ expression was observed among the mutants analyzed (Figure 3B). Significantly, the deletion derivatives showed the same susceptibility to MobC repression as the original 417-bp fragment present in PJSB7.9 (Figure 3B). This indicated that DR1 (IR), DR2(IR), IR1 and the left arm of IR2 (IR2a) were not required for MobC to exert its regulatory effect. To exclude the possibility that one arm of IR2 could suffice for MobC binding, IR2b was mutated in a truncated promoter fragment of $100 \mathrm{bp}$ (pJSB7.14) by site-directed PCR mutagenesis. The deletion of the IR2a arm and multiple substitutions in the remaining IR2b arm had no significant influence on MobC repression.

The above analysis of truncated mobCp fragments localized the MobC-binding site to a 100-bp region encompassing the promoter sequences and two palindromic motifs IR3 and IR4. Both IR3 and IR4 partly overlap the predicted promoter sequence (Figure 1B) hence only limited modifications could be introduced into this region without affecting the promoter integrity (Figure 4A). The PCR-based mutagenesis was performed on the pUC18 [43] derivative with the whole 417-bp insert (pJSB2.9), since the truncated $m o b C p$ regions exhibited variations in the transcriptional activity. The mutated inserts were recloned into the promoter-probe vector pPT01 and tested for mobCp activity and sensitivity to the MobC, produced from pJSB5.1 in trans. Nevertheless, even those few nucleotide substitutions led to a decrease of the $m o b C p$ 


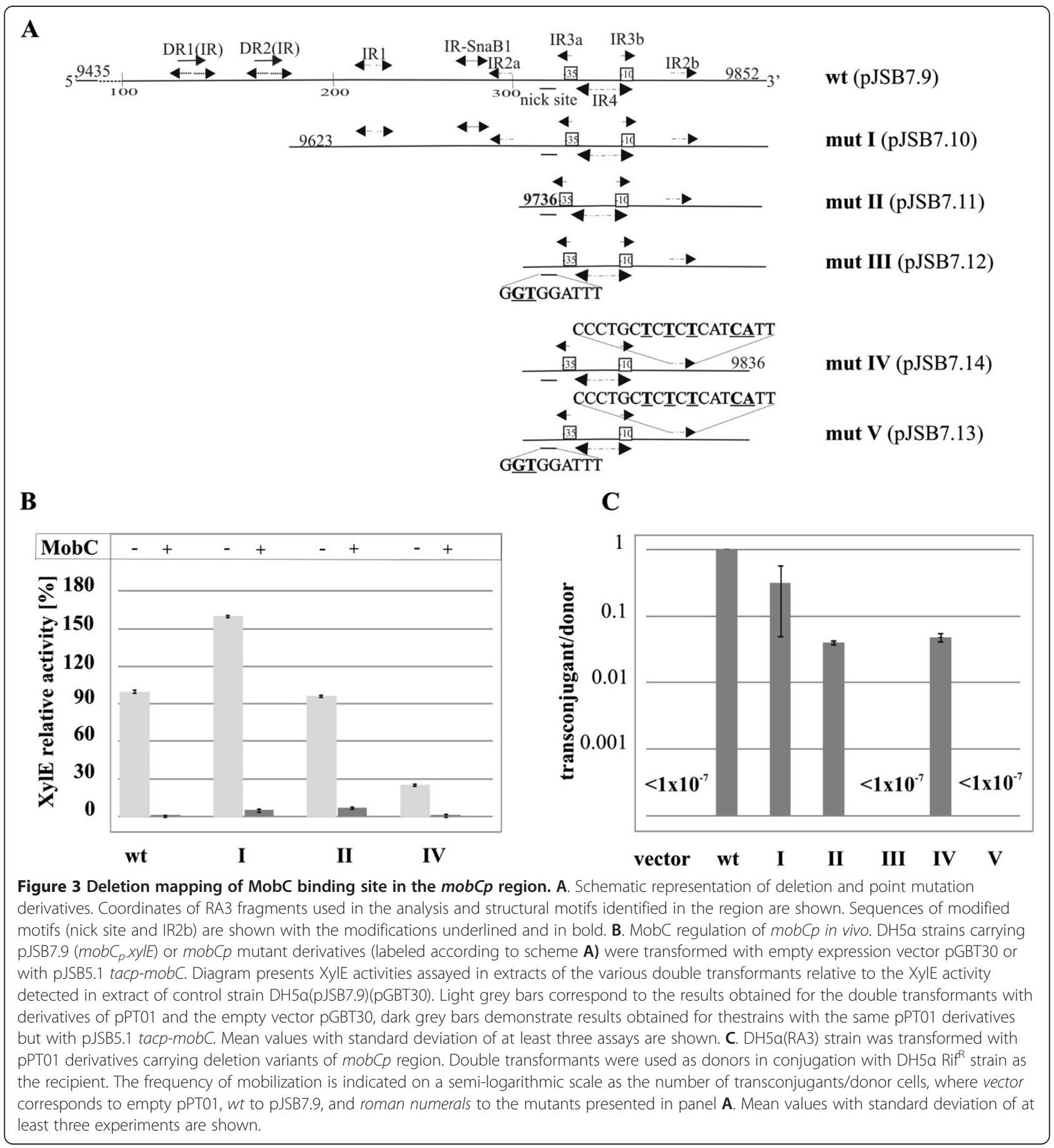

activity (up to 5 -fold) in some of the constructs. MobC repression was unaffected when IR3a was modified as in pJSB7.18 (GCAATT $\rightarrow$ TAGATT) whereas substitutions in IR4 (pJSB7.15, pJSB7.16 and pJSB7.17) led to a decrease in repression by MobC (Figure 4C).

When one arm of the IR4 palindrome was modified $(\mathrm{AGCGTCTG} \uparrow \mathrm{CCGCCGCT} \rightarrow \mathrm{ACGCAGAC} \uparrow \mathrm{CCGCCGCT})$ to give variant mutVI (pJSB7.15), the promoter became hardly sensitive to MobC repression (repression index
1.5), strongly suggesting that IR4 is the operator for MobC and that efficient MobC binding requires the intact palindrome (Figure 4A and C).

IR4 is not a perfect palindrome, having two pairs of noncomplementary nucleotides (AGCGTCTG $\uparrow$ CCGCCGCT). To create a perfect palindromic sequence either left or right arm of the IR4 was modified by site-directed mutagenesis. Version of IR4 (AGCGGCGG $\uparrow C C G C C G C T$ ) in mutVII (pJSB7.16) had a higher content of GC pairs whereas 
A
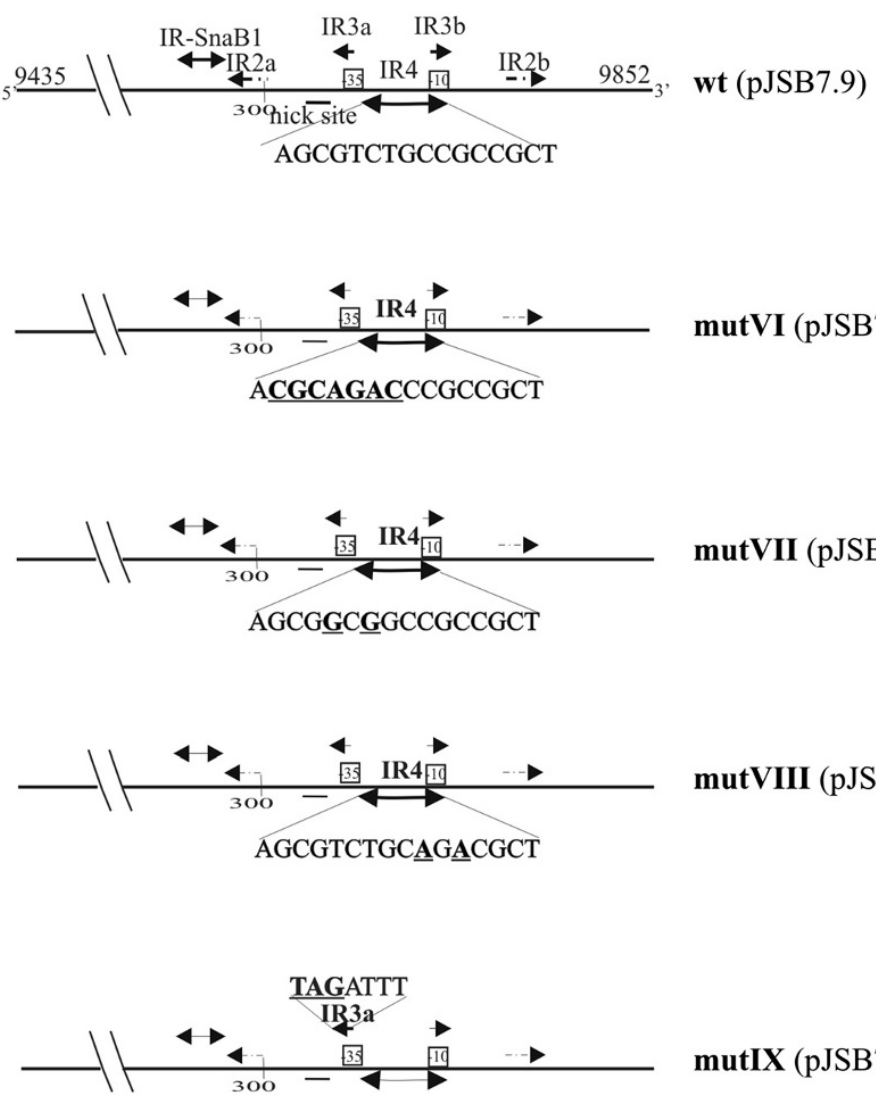

C

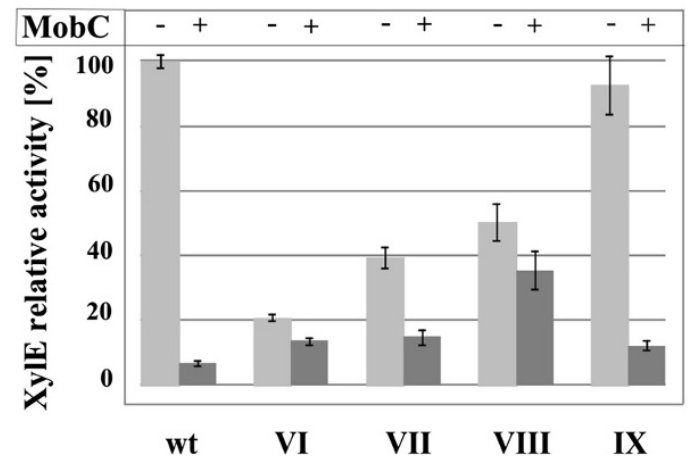

Figure 4 (See legend on next page.)

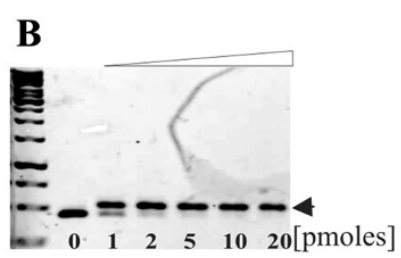

mutVI (pJSB7.15)

mutVII (pJSB7.16)

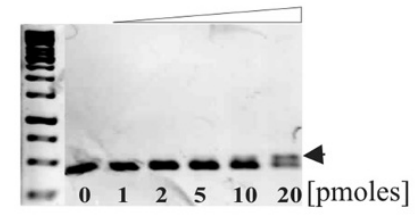

mutVIII (pJSB7.17)

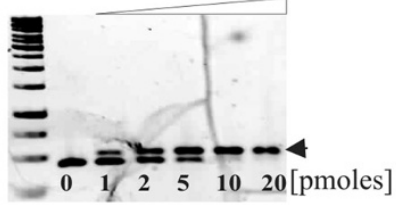

mutIX (pJSB7.18)

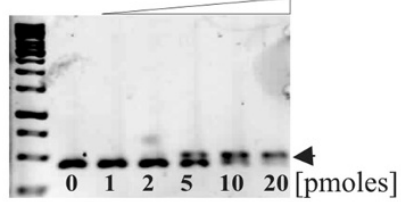

non specific DNA $(k l c A p)$
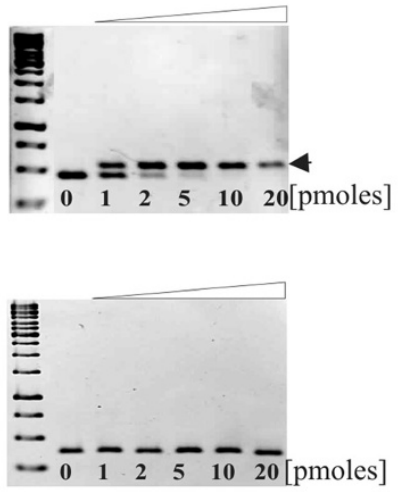

D

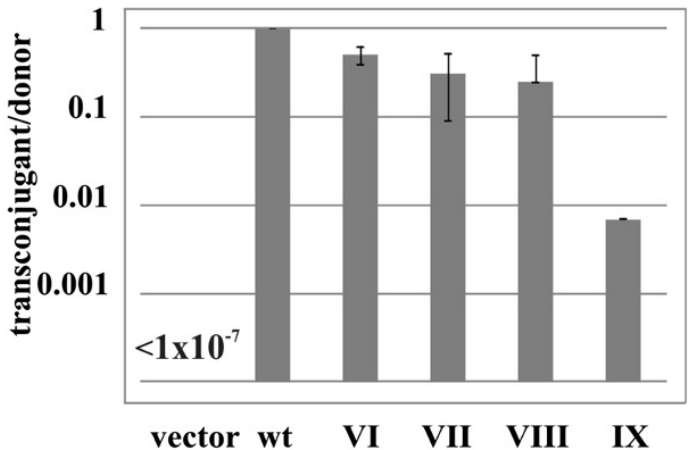


(See figure on previous page.)

Figure 4 Modifications of MobC operator. A. Scheme of PCR site-directed modifications in mobCp region. Sequences of modified motifs (IR3 and IR4) are shown with the modifications underlined and in bold. Mutant alleles (mutVI-IX) were PCR-amplified and analyzed in EMSA (panel B), cloned into promoter-probe vector pPT01 upstream of xylE cassette (panel $\mathbf{C}$ and D). B. DNA binding activity of MobC in vitro. Two picomoles of 417-bp DNA fragments amplified by PCR on templates shown in panel $\mathbf{A}$ were incubated with 0 to 20 picomoles of His-tagged MobC in $20 \mu \mathrm{l}$ of binding buffer at $37^{\circ} \mathrm{C}$ for 15 minutes. The complexes were separated on 1.2\% agarose gels run in 1xTBE and visualized by ethidium bromide staining. The bottom panel refers to EMSA of MobC with unspecific DNA fragment, PCR-amplified kICAp of RA3 (coordinates 2336-2704 nt). C. MobC regulation of mobCp derivatives in vivo. DH5a strains carrying pJSB7.9 (wt 417 bp fragment) or its mutant derivatives (labeled according to panel A) were transformed with empty vector pGBT30 (light grey bars) or pJSB5.1 tacp-mobC (dark grey bars). Diagram presents XyIE activities in extracts of the double transformants relative to the XylE activity detected in extract of DH5a(pJSB7.9)(pGBT30) strain. Mean values with standard deviation of at least three assays are shown. D. DH5a (RA3) strain was transformed with pPT01 derivatives carrying substitution mutant variants of mobCp region. Double transformants were used as donors in conjugation with DH5a Rif $^{R}$ strain as the recipient. The frequency of mobilization is indicated on a semi-logarithmic scale as the number of transconjugants/donor cell, where vector corresponds to empty pPT01, wt to pJSB7.9 $($ mobCp-xylE), and roman numerals to the mutants presented in panel $\mathbf{A}$. Mean values with standard deviation of at least three experiments are shown.

version of IR4 (AGCGTCTG $\uparrow A$ GACGCT) in mutVIII (pJSB7.17) had a higher content of AT pairs than the wt sequence. None of these perfect palindromes was fully effective in MobC binding. In comparison to the 20-fold repression observed for the native non-perfect IR4, the repression index was 1.4 and 2.6 , when the mutated versions of IR4 mutVIII or mutVII, respectively, were introduced into mobCp (Figure 4C).

\section{DNA binding by MobC}

The $m o b C$ orf was cloned under $T 7 p$ into pET28a to give pJSB6.1. $\mathrm{His}_{6}$-MobC over-produced in BL21(DE3) (pJSB6.1) strain was purified by affinity chromatography and used in the Electrophoretic Mobility Shift Assays (EMSA) with wt mobCp fragment and its mutated versions.

MobC was able to bind and retard efficiently the 417bp fragment comprising wt $m o b C p$ whereas no MobC binding was observed when an unspecific DNA fragment was used (Figure 4B). The affinity of MobC towards its operator $\mathrm{O}_{\mathrm{M}}$ in the mobCp region estimated by $\mathrm{K}_{\text {app }}$ ( $K_{\text {apparent }}$ - the protein concentration at which $50 \%$ of the DNA fragments were shifted) varied in the range of 30-50 nM for different protein preparations.

DNA binding studies performed with four mutated $m o b C p$ fragments showed that modification of IR3 (mutIX) had no effect on the MobC binding ( $K_{\text {app }} \sim 40$ $\mathrm{nM})$. The binding affinity of MobC to fragments with the three versions of IR4 depended on the type of nucleotide substitution (Figure $4 \mathrm{~B}$ and $\mathrm{C}$ ). The multiple nucleotide changes in one arm of IR4 (mutVI present in pJSB7.15) drastically decreased the MobC affinity ( $K_{\text {app }}>$ $0.8 \mu \mathrm{M})$. Creating a perfect palindromic sequence by replacing two Ts with two Gs in the left arm of $\mathrm{O}_{M}$ (mutVII) increased the $K_{\text {app }}$ to $100 \mathrm{nM}$. The fragment with the perfect palindromic sequence mutVIII with two Cs substituted by As in the right arm (pJSB7.17) was bound by MobC with an approximately 10-fold lower affinity ( $\left.\mathrm{K}_{\mathrm{app}} \sim 400 \mathrm{nM}\right)$ than the wt sequence. The data of in vitro experiments correlated with the decreased index of repression in vivo and confirmed that IR4 plays a vital role in MobC binding and as such it was designated $\mathrm{O}_{M}$ operator for MobC.

\section{Dimerization ability of MobC}

To analyze the ability of MobC to self-interact in vivo and to define the role of the C-terminus in this process, two 3'-end deletion mutant derivatives mobC1-129 and mobC1-155 were constructed, encoding N-terminal 129 and 155 amino acids, respectively (Figure 2B). The $m o b C$ orf and its mutant derivatives were cloned into the vectors of bacterial two-hybrid system BACTH [31]. The wt $m o b C$ was inserted into two pairs of BACTH vectors to fuse MobC translationally with Cya fragments at the Nor the C-terminus. In two-hybrid tests MobC demonstrated a dimerization ability independently of which end of the protein was linked to the Cya fragments. The two truncated forms of MobC differed in their ability to self-interact in the BACTH system (Figure 5A). MobC1155 was not impaired in the dimerization in vivo whereas MobC1-129 lacked the self-interaction ability, suggesting that the C-terminal residues between 130 and 155 are important for this interaction (Figure 2B).

The mobC1-129 and mobC1-155 orfs were cloned under $T 7 p$ into pET28a to give pJSB6.2 and pJSB6.30, respectively. The $\mathrm{His}_{6}-\mathrm{MobC1} 129$ and $\mathrm{His}_{6}-\mathrm{MobC1} 155$ were over-produced in BL21(DE3) transformants and purified by affinity chromatography according to the protocol used for WT MobC.

Glutaraldehyde cross-linking confirmed the ability of WT MobC and MobC1-155 to form dimers, trimers and tetramers in solution (Figure 5B) at low concentrations of the protein and the cross-linking agent. MobC1-129 only formed dimers under the highest glutaraldehyde concentration used. This suggests that the C-terminus of MobC RA3 is not absolutely required for dimerization, but its presence probably stabilizes the dimers (interactions between MobC1-129 and WT MobC were too weak to be detected in the BACTH system) and probably facilitates the formation of higher oligomers. To 


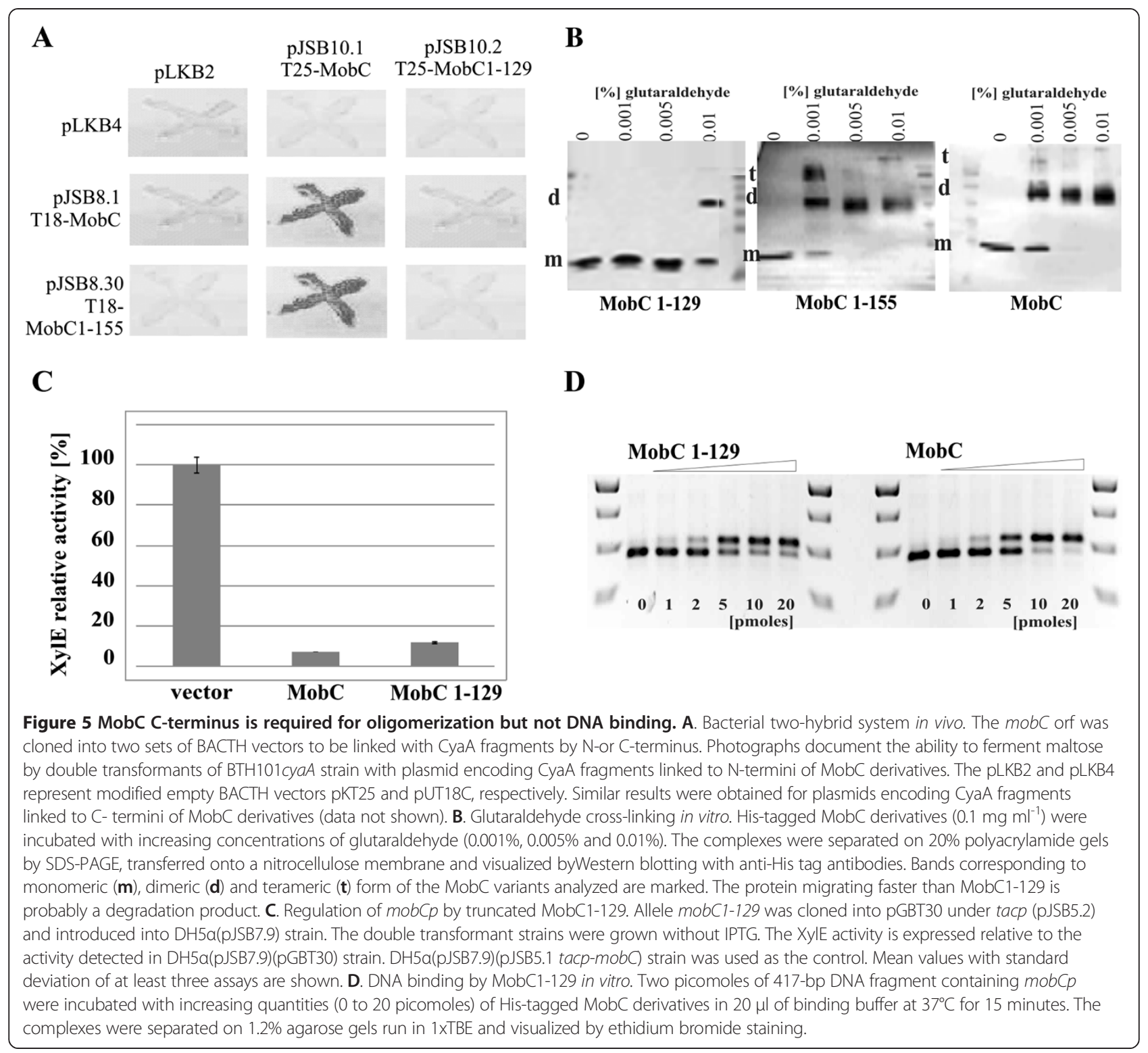

verify these results the gel filtration chromatography was used to analyze the oligomeric state of purified proteins WT MobC and MobC1-129. The molecular weights of both proteins in their monomeric state were estimated by mass spectrometry as $24 \mathrm{kDa}$ and $18 \mathrm{kDa}$, respectively. The gel filtration profile of WT MobC indicated the major form of $49 \mathrm{kDa}$ (Additional file 3) suggesting that MobC exists as a dimer in solution. No higher oligomers of MobC were detected under used conditions. The gel filtration profile of MobC1-129 confirmed its ability to form dimers since the major peak corresponded to protein of $42 \mathrm{kDa}$.

Purified His-tagged MobC1-129 was used in EMSA with wt $m o b C p$ fragment and found to bind DNA with a similar affinity as WT MobC (Figure 5D).

To confirm the ability of MobC1-129 to bind DNA in vivo the deletion variant mobC1-129 was cloned under tacp into the pGBT30 expression vector (pJSB5.2). Using the $m o b C p-x y l E$ transcriptional fusion in the two-plasmid regulatory system we found that MobC1-129 retained activity of a potent repressor. Strong repression of $\mathrm{mobCp}$ $x y l E$ was observed even at a low concentration of the truncated MobC1-129 in trans (when no IPTG inducer was added), similarly to the effect of WT MobC. This suggests that the observed defect in dimerization (BACTH) does not affect the repressor function of MobC1-129 in vivo (Figure $5 \mathrm{C}$ ).

\section{Frequency of mobilization of modified mobCp fragments by RA3 conjugative system}

In other studied conjugative systems auxiliary proteins are essential as being involved in relaxosome formation, specific recognition of nick site by relaxase and oriT 
processing [5]. To check if binding of MobC to ori $T_{\mathrm{RA} 3}$ affects basic relaxase function, conjugative transfer mobilization experiments with plasmids carrying mobCp derivatives were performed.

The pPT01 plasmid with a wt mobCp fragment inserted (pJSB7.9) was introduced into the DH5 $\alpha(\mathrm{RA} 3)$ strain to create a donor strain that could transfer RA3 by conjugation and/or mobilize pJSB7.9 into the recipient strain DH5 $\alpha$ Rif $^{\mathrm{R}}$. The frequency of RA3 self-transfer was estimated to be close to $100 \%$ (one transconjugant per donor cell), the same as the mobilization frequency of pJSB7.9 by RA3. Similar experiments performed with the truncated derivatives of $m o b C p$ demonstrated that deletions of the upstream sequences decreased the mobilization frequency 5 to 20 -fold (Figure 3C).

In the course of this analysis we also constructed mutant derivatives with two nucleotide substitutions at the putative nick site (mutIII and mutV as shown on Figure 3A). pPT01 derivatives carrying these alleles (pJSB7.12 and pJSB7.13) were not transferable by RA3 to the recipient strain under the conditions used (estimated frequency of transfer $<1$ per $10^{7}$ donor cells). These results confirm the vital role of these two nucleotides in the recognition/nicking of oriT (Figure 3C).

A 100-fold decrease in the mobilization frequency was observed for a derivative of the 417-bp mobCp-oriT fragment with several substitutions in the left arm of IR3 (pJSB7.18, mutIX) (Figure 4D). Further studies will be necessary to define the role of these nucleotides in the binding of relaxase to oriT.

Most significantly, modifications of $\mathrm{O}_{M}$ in a full-length mobCp-oriT fragment (417 bp) did not drastically affect the mobilization frequency of tested pPT01 derivatives (Figure 4D). This strongly implicates that the MobC binding to DNA at $\mathrm{O}_{M}$, although important for the transcriptional control of $m o b C p$, is not strictly required for the conjugation process. It was further confirmed in the studies described in the next paragraph (for pJSB2.57).

Although the data clearly demonstrated that MobCDNA interactions are not important in the mobilization experiments (when autoregulatory function of MobC is irrelevant) it did not exclude the possibility of MobC acting as the auxiliary protein through direct interactions with the relaxase or other components of the conjugative machinery.

\section{Influence of MobC on conjugation frequency of vectors with Tra module of RA3}

The conjugative transfer module of RA3 (coordinates 9437-22925 nt) was previously cloned into the high-copynumber plasmid pBGS18 [41] together with korC gene (coordinates 3391-3705 nt) encoding RA3 global transcriptional regulator $[42,51]$. Such construct pJSB1.24 was capable of self-transmission [42] at the frequency comparable with parental RA3 (approximately one transconjugant per donor cell).

To analyze the significance of MobC for the efficiency of the conjugation process it was necessary to separate its autorepressor function from the potential conjugative transfer accessory role. The conjugative transfer region (and $k o r C$ ) was re-cloned into pUC18 and the promoter region of mobC-nic operon was replaced by $l a c I^{\mathrm{q}}$-tacp regulatory region from pGBT30 [35]. The $45 \mathrm{nt}$ fragment encompassing ori $T_{\mathrm{RA} 3}$ but with $\mathrm{O}_{\mathrm{M}}$ and part of the mobCp sequences deleted (coordinates 9722-9766 nt, Figure 1B), designated ori $_{45}$, was inserted into pUC18 derivative with the conjugative transfer module and mobC-nic under control of tacp to get the pJSB2.57 (Figure 6B). The frequency of self-transmission of pJSB2.57 was very high, comparable to RA3 (Figure 6C) confirming that a/ level of expression of mobC-nic operon from the uninduced tacp is adequate for efficient conjugation process, $\mathbf{b} /$ the lack of MobC binding site $\mathrm{O}_{M}$ has no influence on the transfer efficiency.

To verify inability of MobC to bind to ori $T_{45}$ two oligonucleotides $61 \mathrm{nt}$ and $45 \mathrm{nt}$ long were cloned into pUC18 vector to obtain pJSB2.43 and pJSB2.44, respectively. The 61 nt fragment encompassed oriT, IR3a and IR4 $\left(\mathrm{O}_{\mathrm{M}}\right)$ sequences (Figure 1B) whereas $45 \mathrm{nt}$ fragment (as described above) was deprived of $\mathrm{O}_{\mathrm{M}}$. Plasmid DNAs were cut by PvuII in two fragments. The smaller fragments of both plasmid DNA: $383 \mathrm{nt}$ and $367 \mathrm{nt}$ contained inserts of 61 nt and $45 \mathrm{nt}$, respectively. In the EMSA experiment the 45 nt sequence, deprived of $\mathrm{O}_{M}$, has not been recognized by MobC, the protein has bound specifically only to the fragment of $383 \mathrm{nt}$ with $61 \mathrm{nt}$ of oriT inserted (Figure 6A).

To check if MobC itself plays a role in the conjugative transfer the pJSB2.58 was constructed, the variant of pJSB2.57 in which the mobC gene was absent and only nic was expressed from the tacp (Figure 6B). The 1000-fold decrease in the frequency of self-transmission of pJSB2.58 indicated that MobC indeed acts as the conjugative transfer auxiliary protein. The strain DH5 $\alpha($ JJSB2.58) was transformed with medium-copy number expression plasmid pJSB4.1 (tacp-mobC) and the double transformant was used as the donor strain in the conjugation. The high frequency of self-transmission of pJSB2.58 was restored (Figure 6C). Similar effect of restoration was achieved when lack of $m o b C$ in pJSB2.58 was complemented by tacpmobC1-129 expressed in trans in the DH5 $\alpha$ (pJSB2.58) (pJSB4.2) strain. It indicated that despite dimerization/ oligomerization deficiency MobC1-129 is not only a potent auto-repressor but also its function in enhancing the frequency of transfer is unaltered.

\section{Discussion}

The RA3 plasmid, the archetype of IncU group, has a mosaic-modular structure with the conjugative system 
A

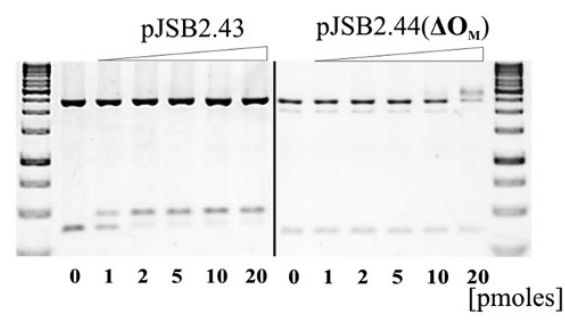

B
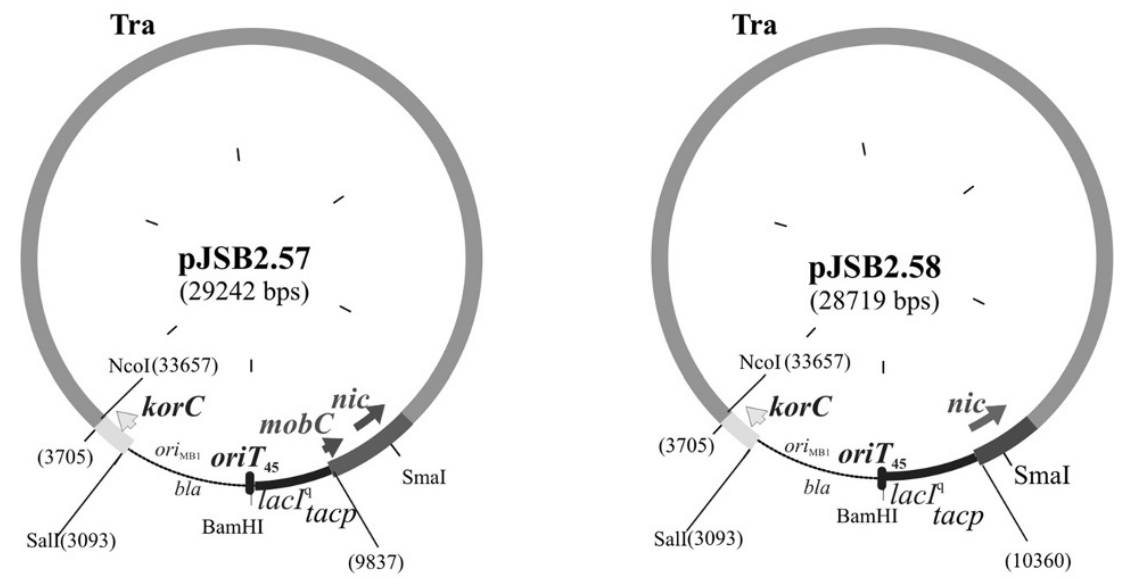

C

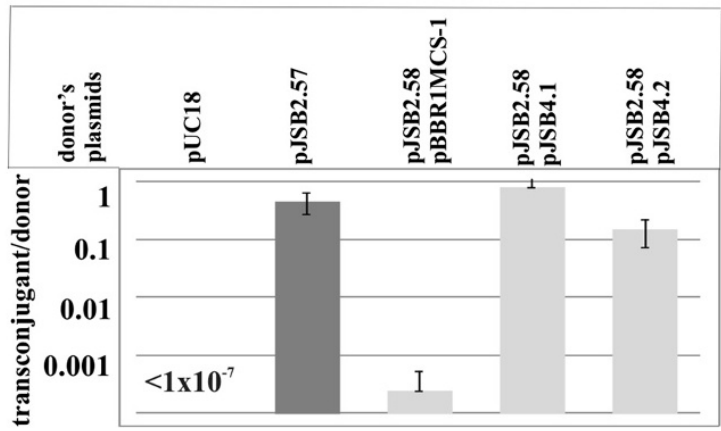

Figure 6 MobC influence on efficiency of self-transmission of plasmids with RA3 conjugative module. A. DNA binding of MobC in vitro. Plasmid DNAs of pJSB2.43 (61 nt insert with $\mathrm{O}_{\mathrm{M}}$ ) and pJSB2.44 (45 nt insert without $\mathrm{O}_{\mathrm{M}}$ ) were digested by Pvull and incubated with different amounts of $\mathrm{His}_{6}-\mathrm{MobC}$ in $20 \mu \mathrm{l}$ of binding buffer at $37^{\circ} \mathrm{C}$ for 15 minutes $(0-20$ pmoles). The complexes were separated on $1 \%$ agarose gels run in 1xTBE and visualized by ethidium bromide staining. Fragment $2364 \mathrm{nt}$ corresponds to "unspecific" vector DNA, small fragments in both plasmid DNAs contain oriT $T_{\text {RA3 }}$ with $\mathrm{O}_{\mathrm{M}}$ (383 nt) or without MobC binding site (367 nt). B. Schematic presentation of tested vectors carrying the RA3 conjugative module with mobCp substituted by lacla tacp (no $\mathrm{O}_{M}$ ). Dark grey regions correspond to the contiguous Tra $\mathrm{RA3}$ region and light grey parts indicate korCp-korC fragment from RA3 maintenance module. Black regions show mobC-nic or nic genes under tac promoter control. The 45 nt oriT $T_{\text {RA3 }}$ is indicated by black rectangular. Restriction sites used during construction of pJSB2.57 and pJSB2.58 are listed. Numbers in brackets correspond to RA3 coordinates. C. The effect of MobC presence on the conjugation frequency. The strains: DH5a(pUC18), DH5a(pJSB2.57), DH5a(pJSB2.58)(pBBR1MCS-1), DH5a(pJSB2.58)(pJSB4.1) and DH5a(pJSB2.58)(pJSB4.2) served as donors in conjugation with the recipient strain DH5a Rif ${ }^{\mathrm{P}}$. Conjugation frequency is indicated on the semilogarithmic scale as the number of transconjugants per total number of donor cells. Mean values with standard deviation of at least three experiments are shown.

similar to that found in many promiscuous environmental plasmids, some of them recently classified into the new group designated PromA [25].
We have initiated an experimental dissection of the IncU conjugative transfer system to understand the reasons of the wide spreading of such modules among 
conjugative plasmids of different incompatibility groups and their high efficiency of transfer between a broad range of hosts. In this study we concentrated our efforts on a functional analysis of the MobC protein (encoded by the first gene of the conjugative module) and its interactions with DNA.

The data demonstrates that MobC is a potent repressor of $m o b C p$ and as such controls the level of relaxase production. Using different experimental approaches we identified the MobC-binding site $\mathrm{O}_{M}$ at a region overlapping the mobC promoter. This non-perfect palindromic sequence AGCGTCTG $\uparrow C C G C C G C T$ is recognized by MobC in vitro with a high affinity. Attempts to improve the $\mathrm{O}_{M}$ by creating a perfect palindrome failed, showing clearly that the existing slightly imperfect configuration is optimal for MobC binding.

Purified $\mathrm{His}_{6}$-MobC forms mainly dimers as it was shown by gel filtration chromatography in these solution conditions. Some auxiliary proteins e.g. TrwA of R388 exist as tetramers in solution [28] others like MbeC of ColE1 plasmid are mainly dimeric [13]. To localize dimerization domain we analyzed C-terminally truncated MobC derivatives. Deletion of C-terminal 47 amino acids (MobC1-129) but not 21 amino acids (MobC1-155) impaired selfinteractions in vivo when tested in BACTH system. The purified MobC1-129 formed dimers in solution as shown by use of the molecular sieve. The intact MobC had an ability to form trimers and tetramers in the presence of the cross-linking agent whereas MobC1-129 formed only dimers under these conditions. So far the function of the higher order forms of MobC is not clear.

MobC1-129 binds efficiently to the operator sequence and represses mobCp in vivo to the same extent as does the WT MobC. It means that the N-terminal part is sufficient for specific DNA binding and a weak dimerization whereas the $\mathrm{C}$-terminus is required to stabilize the dimers.

Deletion of C-terminal 47 amino acids removes part of the conserved "bacterial mobilization motif" (Figure 2A; Additional file 2). The role of this motif in interactions of the auxiliary proteins with the conjugation machinery has been implicated [14,30]. Truncated MobC1-129 fully complemented the role of WT MobC in enhancing the conjugative transfer. Hence whether MobC assists the relaxase $\mathrm{Nic}$ in its functions or stimulates the coupling protein VirD4 (or any other protein) it does not require the intact motif. Direct interactions have been detected using the BACTH system neither between MobC and Nic nor MobC and VirD4 so far (data not shown).

MobC differs from known conjugative transfer auxilliary proteins since its binding to DNA close to oriT seems not to be strictly required for the relaxase action at oriT. All mobilizable plasmids with the $\mathrm{O}_{M}$ modified in such a way as to abolish or severely impair MobC binding (confirmed by a decreased repression in vivo and lower DNA binding affinity in vitro) demonstrated highly similar mobilization frequency when compared to plasmid pJSB7.9 with a wt fragment inserted. Moreover, the frequency of self-transmission of plasmid pJSB2.57, carrying RA3 conjugative module but with oriT deprived of $\mathrm{O}_{\mathrm{M}}$ (Figure 6) has not been even slightly affected.

The absence of MobC did not stop the self-transmission of pJSB2.58, although it led to 1000-fold decrease in the transfer efficiency in comparison to pISB2.57. Whereas significant, it is a much less pronounced effect than observed in other studied plasmids when deprived of the auxiliary proteins $[13,19,29]$.

The functionality of oriT in the centromere-like region of the partition operon has been shown experimentally for RA3 of IncU [21]. There, the nick site precedes the transcription start point for $m o b C$ by $50 \mathrm{nt}$, and substitutions of two nucleotides in this sequence (pJSB7.12 and pJSB7.13) abolish the mobilization capacity of a test plasmid (with the oriT-mobCp fragment inserted) by the RA3 conjugation system. Our analysis of mutants with nucleotide substitutions adjacent to the nick site has revealed the importance of one arm of a short palindromic sequence designated IR3 for the processing of oriT (mobilization frequency). Further studies are required to understand the role of this motif in the transfer efficiency.

Plasmids of the IncU and PromA incompatibility groups share the transcriptional organization of at least two putative conjugative transfer operons. The location of the oriT sequence in the promoter regions of $m o b C$ nic and their counterparts in other plasmids is at the border of conjugative transfer modules and the partition operons $[21,25,43-46]$, however the nick sites identified in silico in PromA representatives do not precede but rather overlap the predicted -35 sequences of promoters for the mobC homologs: orf15 of pSB102, orf21 of pIPO2, orf57 of pMOL58, mobC of pTer331 and mobC of pMRAD02 (Figure 7).

Interestingly, the conservation of the promoter regions of the mobC counterparts in the IncU and PromA plasmids has been lost around the sequence of the identified $\mathrm{O}_{\mathrm{M}}$ in RA3 (Figure 7). In the PromA plasmids there are palindromic sequences overlapping or located downstream of a predicted -10 motif, presumably forming the binding site for the MobC homologs.

The role of the other structural motifs in the mobCp-oriT region of RA3 (Figure 1B) remains unknown but a possibility of tertiary cruciform structure formation (predicted by program Geneious 6.1.3) seems very attractive especially for the two inverted repeats IR2 and IR3 with arms separated by $75 \mathrm{nt}$ and $26 \mathrm{nt}$, respectively (Additional file 4).

One of these palindromes, the GC-rich IR2, is highly conserved between RA3 and three out of five PromA representatives not only in the primary sequence but 


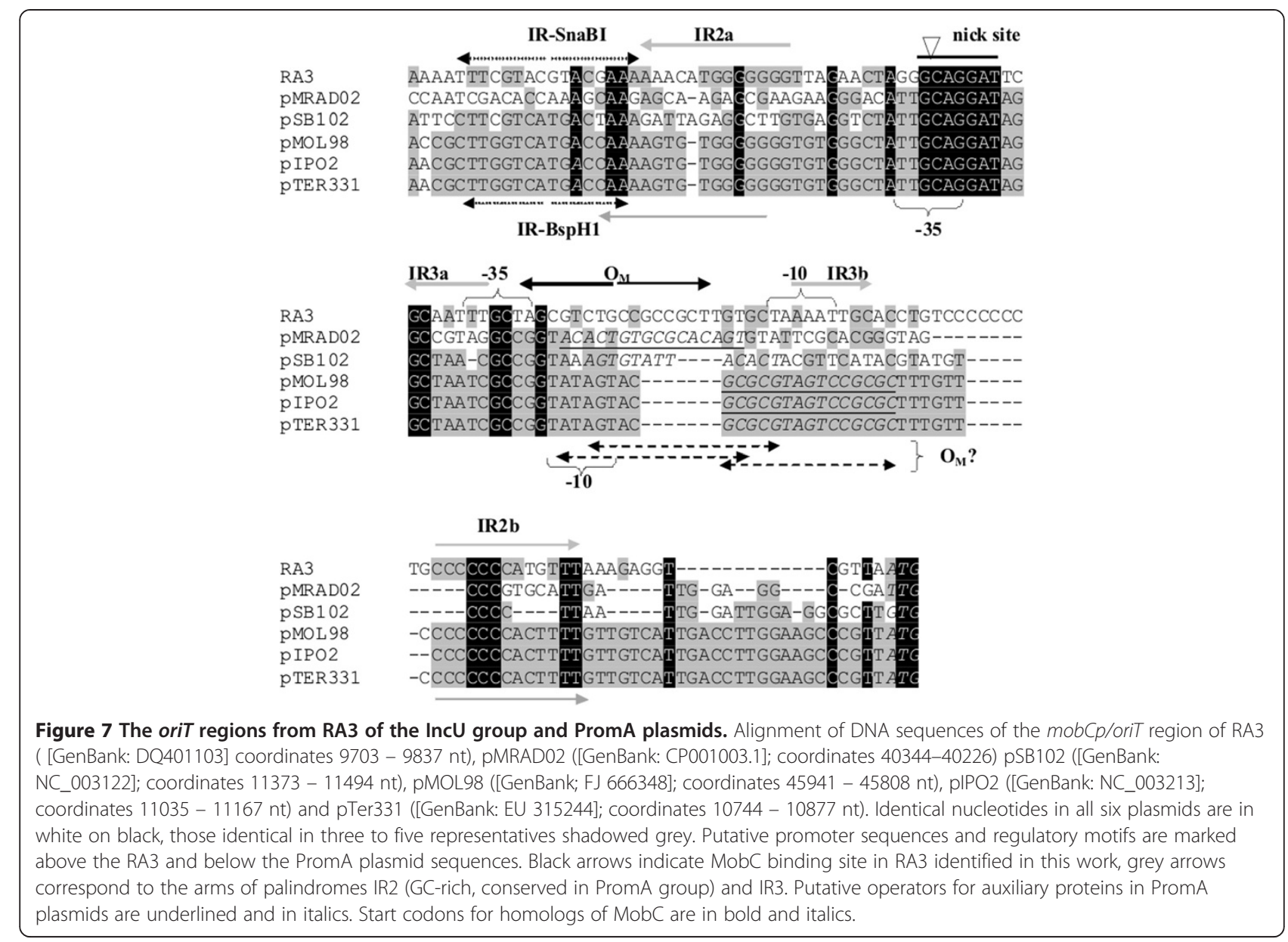

also in the position of the arms encompassing promoter sequences, putative $\mathrm{O}_{M}$ and nick sites (Figure 7). Our future studies will be aimed at understanding the functions of these motifs and the interplay between Nic and MobC (relaxosome), IncC and KorB (segrosome) and transcriptional machinery at a potentially highlystructured parS-oriT-mobCp region that must accommodate all these complexes.

\section{Conclusions}

In this work we have demonstrated that MobC of RA3 plasmid acts as an auxiliary transfer protein of dual function. It autoregulates the expression of $m o b C p$ controlling the level of relaxase production. It binds to DNA recognizing an imperfect palindromic sequence $\left(\mathrm{O}_{M}\right)$ overlapping the promoter motifs. DNA binding domain is localized in the N-terminal part of the protein. The Cterminus participates in the stabilization of oligomeric forms. Besides its role as the transcriptional repressor MobC stimulates the frequency of conjugation process. For this activity MobC binding in the oriT region is not required. Future studies should establish whether MobC stimulates relaxosome formation, activity of relaxase or affects other stages of the conjugation process (e.g. interactions with coupling protein). The role of the structural motifs identified in the oriT of IncU, and conserved in putative oriT regions of PromA plasmids, awaits elucidation.

\section{Additional files}

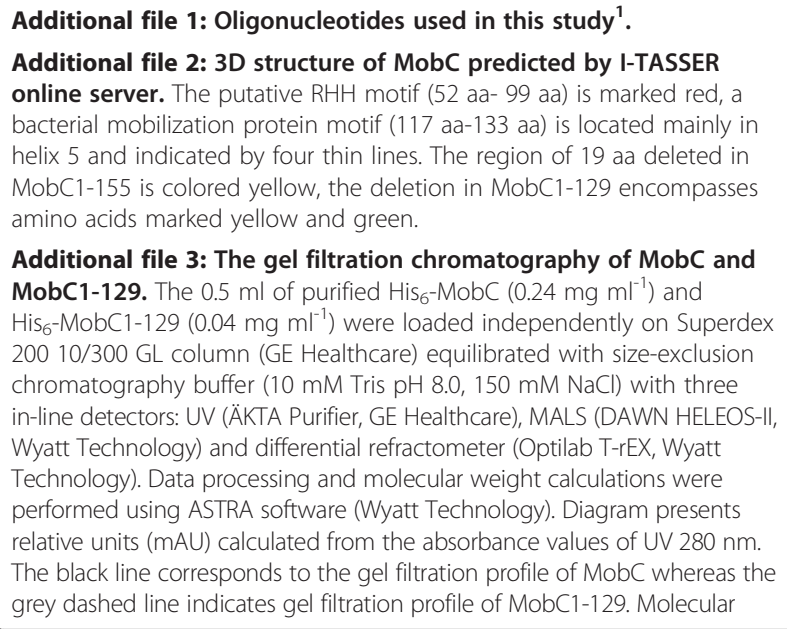

Additional file 3: The gel filtration chromatography of MobC and MobC1-129. The $0.5 \mathrm{ml}$ of purified $\mathrm{His}_{6}-\mathrm{MobC}\left(0.24 \mathrm{mg} \mathrm{ml}^{-1}\right)$ and $\mathrm{His}_{6}-\mathrm{MobC1}-129$ (0.04 $\left.\mathrm{mg} \mathrm{ml}^{-1}\right)$ were loaded independently on Superdex 200 10/300 GL column (GE Healthcare) equilibrated with size-exclusion chromatography buffer (10 mM Tris pH 8.0, $150 \mathrm{mM} \mathrm{NaCl}$ ) with three in-line detectors: UV (ÄKTA Purifier, GE Healthcare), MALS (DAWN HELEOS-II, Wyatt Technology) and differential refractometer (Optilab T-rEX, Wyatt Technology). Data processing and molecular weight calculations were performed using ASTRA software (Wyatt Technology). Diagram presents relative units (mAU) calculated from the absorbance values of UV $280 \mathrm{~nm}$. The black line corresponds to the gel filtration profile of MobC whereas the grey dashed line indicates gel filtration profile of MobC1-129. Molecular 
weight of complexes His $s_{6}-\mathrm{MobC}$ and His $s_{6}-\mathrm{MobC1}$-129 are $49 \mathrm{kDa}$ and 42 $\mathrm{kDa}$ respectively. Data from mass spectrometry show that molecular weight of $\mathrm{His}_{6}-\mathrm{MobC}$ monomer is $24 \mathrm{kDa}$ and His ${ }_{6}-\mathrm{MobC1}-129$ monomer is $18 \mathrm{kDa}$ (Technical specification: Spectrometer Synapt G2 MS (Waters), LC system nanoACQUITY (Waters), Trap Column ACQUITY UPLC PrST C4 VanGuard, Pre-column 300A, $1.7 \mu \mathrm{m}, 2.1 \mathrm{~mm}, 5 \mathrm{~mm}$ ).

Additional file 4: Single stranded DNA folding of orit $T_{\mathrm{RA}}$ region and its mutant derivatives. The MobC binding site (IR4) is shadowed in grey, the -35 and -10 sequences of mobCp are circled in pink, the nick site is circled in red with arrows pointing the site of cleavage. The IR3 and IR-SnaBI are marked as green or blue rectangles, respectively. The mutants are designated as on Figure 4A. The DNA sequence analyzed by Geneious6.1.3 encompasses region of RA3 between 9560-9840 nt.

\section{Competing interests}

The authors declare that they have no competing interest.

\section{Authors' contributions}

JG performed most part of the experimental work, analyzed the results and helped in writing the manuscript. AK participated in the initial experiments and helped in writing the manuscript. GJB designed and coordinated the study, participated in analysis of the results and wrote the manuscript. All authors read and approved the final version.

\section{Acknowledgements}

We would like to thank Andrzej Dziembowski PhD and Mariusz CzarnockiCieciura MSc from the Laboratory of RNA Biology and Functional Genomics IBB PAS for help with SEC-MALS analysis.

This work was funded by grant PBZ-MNiSW-04/1/2007 and partially by grant MNiSW 7793/B/P01/2011/40 and NCN 2011/03/B/NZ1/06540.

\section{Author details}

'Institute of Biochemistry and Biophysics, Department of Microbial Biochemistry, PAS, 02-106 Warsaw, Poland. ${ }^{2}$ Present address: Warsaw University of Technology, Faculty of Chemistry, Warsaw, Poland.

Received: 15 May 2014 Accepted: 19 August 2014

Published: 4 September 2014

\section{References}

1. Christie PJ, Cascales E: Structural and dynamic properties of bacterial Type IV secretion systems. Mol Membr Biol 2005, 22:51-61.

2. Schröder G, Lanka E: The mating pair formation system of conjugative plasmids - A versatile secretion machinery for transfer of proteins and DNA. Plasmid 2005, 54:1-25.

3. Zechner E, de la Cruz F, Eisenbrandt R, Grahn A, Koraimann G, Lanka E, Muth G, Pansegrau W, Thomas C, Wilkins B, Zatyka M: Conjugative-DNA Transfer Processes. In The Horizontal Gene Pool, Bacterial Plasmids and Gene Spread. Edited by Thomas CM. Amsterdam: Harwood Academic Publishers; 2000:87-174.

4. Guasch A, Lucas M, Moncalian G, Cabezas M, Perez-Luque R, Gomis-Ruth FX, de la Cruz F, Coll M: Recognition and processing of the origin of transfer DNA by conjugative relaxase TrwC. Nat Struct Mol Biol 2003, 10:1002-1010

5. De la Cruz F, Frost LS, Meyer RJ, Zechner EL: Conjugative DNA metabolism in Gram-negative bacteria. FEMS Microbiol Rev 2010, 34:18-40.

6. Trokter M, Felisberto-Rodrigues C, Christie P, Waksman G: Recent advances in the structural and molecular biology of type IV secretion systems. Curr Opin Struct Biol 2014, 27:16-23.

7. Bhatty M, Laverde Gomez JA, Christie PJ: The expanding bacterial type IV secretion lexicon. Res Microbiol 2013, 164:620-639.

8. Fekete RA, Frost $L S$ : Characterizing the DNA contacts and cooperative binding of $\mathrm{F}$ plasmid TraM to its cognate sites at oriT. J Biol Chem 2002, 277:16705-16711.

9. Pansegrau W, Ziegelin G, Lanka E: Covalent association of the tral gene product of plasmid RP4 with the 5'-terminal nucleotide at the relaxation nick site. J Biol Chem 1990, 265:10637-10644.

10. Ragonese H, Haisch D, Villareal E, Choi J-H, Matson SW: The F plasmid-encoded TraM protein stimulates relaxosome-mediated cleavage at oriT through an interaction with Tral. Mol Microbiol 2007, 63:1173-1184.
11. Ziegelin G, Fürste JP, Lanka E: TraJ protein of plasmid RP4 binds to a 19-base pair invert sequence repetition within the transfer origin. J Biol Chem 1989, 264:11989-11994.

12. Ziegelin G, Pansegrau W, Lurz R, Lanka E: TraK protein of conjugative plasmid RP4 forms a specialized nucleoprotein complex with the transfer origin. J Biol Chem 1992, 267:17279-17286.

13. Varsaki A, Moncalian G, del Pilar Garcillan-Barcia M, Drainas C, de la Cruz F: Analysis of ColE1 MbeC unveils an extended ribbon-helix-helix family of nicking accessory proteins. J Bacterio/ 2009, 191:1446-1455.

14. Tato I, Matilla I, Arechaga I, Zunzunegui S, de la Cruz F, Cabezon E: The ATPase activity of the DNA transporter TrwB is modulated by protein TrwA: implications for common assembly mechanism of DNA translocating motor. J Biol Chem 2007, 282:25569-25576.

15. Zatyka M, Jagura-Burdzy G, Thomas CM: Transcriptional and translational control of the genes for the mating pair formation apparatus of promiscuous IncP plasmids. J Bacterio/ 1997, 179:7201-7209.

16. Silverman PM, Sholl A: Effect of traY amber mutations on F-plasmid traY promoter activity in vivo. J Bacteriol 1996, 178:5787-5789.

17. Penfold SS, Simon J, Frost LS: Regulation of the expression of the traM gene of the F sex factor of Escherichia coli. Mol Microbiol 1996, 20:549-558.

18. $\mathrm{Fu}$ YH, Tsai MM, Luo YN, Deonier RC: Deletion analysis of the F plasmid oriT locus. J Bacteriol 1991, 173:1012-1020.

19. Moncalián G, Grandoso G, Llosa M, de la Cruz F: OriT-processing and regulatory roles of TrwA protein in plasmid R388 conjugation. $J$ Mol Biol 1997, 270:188-200.

20. Aoki T, Mitoma Y, Crosa JH: The characterization of a conjugative Rplasmid isolated from Aeromonas salmonicida. Plasmid 1986, 16:213-218.

21. Kulinska A, Czeredys M, Hayes F, Jagura-Burdzy G: Genomic and functional characterization of the modular broad-host-range RA3 plasmid, the archetype of the IncU group. Appl Environ Microbiol 2008, 74:4119-4132.

22. Rhodes G, Parkhill J, Bird C, Ambrose K, Jones MC, Huys G, Swings J, Pickup RW: Complete nucleotide sequence of the conjugative tetracycline resistance plasmid pFBAOT6, a member of a group of IncU plasmids with global ubiquity. App/ Environ Microbiol 2004 70:7497-7510.

23. Yanisch-Perron C, Vieira J, Messing J: Improved M13 phage cloning vectors and host strains: nucleotide sequences of the M13mp18 and pUC19 vectors. Gene 1985, 33:103-119.

24. Brown CJ, Sen D, Yano H, Bauer ML, Rogers LM, Van der Auwera GA Top EM: Diverse broad-host-range plasmids from freshwater carry few accessory genes. Appl Environ Microbiol 2013, 79:7684-7695.

25. Van der Auwera G, Król J, Suzuki H, Foster B, Van Houdt R, Brown C, Mergeay M, Top E: Plasmids captured in C. metallidurans $\mathrm{CH} 34$ : defining the PromA family of broad-host-range plasmids. Antonie Van Leeuwenhoek 2009, 96:193-204.

26. Fürste JP, Pansegrau W, Ziegelin G, Kröger M, Lanka E: Conjugative transfer of promiscuous IncP plasmids: interaction of plasmid-encoded products with the transfer origin. Proc Natl Acad Sci 1989, 86:1771-1775.

27. Bowie JU, Sauer RT: TraY proteins of $F$ and related episomes are members of the Arc and Mnt repressor family. J Mol Biol 1990, 211:5-6.

28. Moncalián G, de la Cruz F: DNA binding properties of protein TrwA, a possible structural variant of the Arc repressor superfamily. Biochim Biophys Acta 2004, 1701:15-23.

29. Guiney DG, Deiss C, Simnad V, Yee L, Pansegrau W, Lanka E: Mutagenesis of the Tra1 core region of RK2 by using Tn5: identification of plasmidspecific transfer genes. J Bacterio/ 1989, 171:4100-4103.

30. Varsaki A, Lamb HK, Eleftheriadou O, Vandera E, Thompson P, Moncalián G de la Cruz F, Hawkins AR, Drainas C: Interaction between relaxase MbeA and accessory protein $\mathrm{MbeC}$ of the conjugally mobilizable plasmid ColE1. FEBS Lett 2012, 586:675-679.

31. Karimova G, Pidoux J, Ullmann A, Ladant D: A bacterial two-hybrid system based on a reconstituted signal transduction pathway. Proc Natl Acad Sci 1998, 95:5752-5756.

32. Kahn MR, Kolter $R$, Thomas CM, Figurski D, Meyer $R$, Remault E, Helinski DR: Plasmid cloning vehicles derived from plasmids ColE1, F, R6K, and RK2. Methods Enzymol 1979, 68:268-280.

33. Sambrook J, Fritsch EF, Maniatis T: Molecular Cloning, a Laboratory Manual. 2nd edition. Cold Spring Harbor, NY: Cold Spring Harbor Press; 1989.

34. Mullis K, Faloona F, Scharf S, Saiki R, Horn G, Erlich H: Specific enzymatic amplification of DNA in vitro: the polymerase chain reaction. Cold Spring Harb Symp Quant Biol 1986, 51:263-273. 
35. Jagura-Burdzy G, Ibbotson JP, Thomas CM: The korF region of broad-hostrange plasmid RK2 encodes two polypeptides with transcriptional repressor activity. J Bacteriol 1991, 173:826-833.

36. Kovach ME, Phillips RW, Elzer PH, Roop RM, Peterson KM: pBBR1MCS: a broad-host-range cloning vector. Biotechniques 1994, 16:800-802.

37. Zukowski MM, Gaffney DF, Speck D, Kauffmann M, Findeli A, Wisecup A Lecocq JP: Chromogenic identification of genetic regulatory signals in Bacillus subtilis based on expression of a cloned Pseudomonas gene. Proc Natl Acad Sci 1983, 80:1101-1105.

38. Bradford MM: A rapid and sensitive method for the quantitation of microgram 486 quantities of protein utilizing the principle of protein-dye binding. Anal Biochem 1976, 72:248-254.

39. Jagura-Burdzy G, Thomas CM: Purification of KorA protein from broad host range plasmid RK2: definition of a hierarchy of KorA operators. J Mol Biol 1995, 253:39-50.

40. Bartosik AA, Lasocki K, Mierzejewska J, Thomas CM, Jagura-Burdzy G: ParB of Pseudomonas aeruginosa: Interactions with its partner ParA and its target parS and specific effects on bacterial growth. J Bacteriol 2004, 186:6983-6998.

41. Spratt BG, Hedge PJ, te Heesen S, Edelman A, Broome-Smith JK: Kanamycinresistant vectors that are analogues of plasmids pUC8, pUC9, pEMBL8 and pEMBL9. Gene 1986, 41:337-342.

42. Bartosik AA, Markowska A, Szarlak J, Kulińska A, Jagura-Burdzy G: Novel broad-host-range vehicles for cloning and shuffling of gene cassettes. J Microbiol Methods 2012, 88:53-62.

43. Kulinska A, Cao Y, Macioszek M, Hayes F, Jagura-Burdzy G: The centromere site of the segregation cassette of broad-host-range plasmid RA3 is located at the border of the maintenance and conjugative transfer modules. Appl Environ Microbiol 2011, 77:2414-2427.

44. Tauch A, Schneiker S, Selbitschka W, Pühler A, van Overbeek LS, Smalla K, Thomas CM, Bailey MJ, Forney LJ, Weightman A, Ceglowski P, Pembroke T, Tietze E, Schröder G, Lanka E, van Elsas JD: The complete nucleotide sequence and environmental distribution of the cryptic, conjugative, broad-host-range plasmid pIPO2 isolated from bacteria of the wheat rhizosphere. Microbiology 2002, 148:1637-1653.

45. Marques MV, da Silva AM, Gomes SL: Genetic organization of plasmid pXF51 from the plant pathogen Xylella fastidiosa. Plasmid 2001, 45:184-199.

46. Schneiker S, Keller M, Dröge M, Lanka E, Pühler A, Selbitschka W: The genetic organization and evolution of the broad host range mercury resistance plasmid pSB102 isolated from a microbial population residing in the rhizosphere of alfalfa. Nucleic Acids Res 2001, 29:5169-5181.

47. Schreiter ER, Drennan CL: Ribbon-helix-helix transcription factors: variations on a theme. Nat Rev Microbiol 2007, 5:710-720.

48. Lum PL, Schildbach JF: Specific DNA recognition by F factor TraY involves B-Sheet residues. J Biol Chem 1999, 274:19644-19648.

49. Yoshida H, Furuya N, Lin Y-J, Güntert P, Komano T, Kainosho M: Structural basis of the role of the NikA ribbon-helix-helix domain in initiating bacterial conjugation. J Mol Biol 2008, 384:690-701.

50. Macartney DP, Williams DR, Stafford T, Thomas CM: Divergence and conservation of the partitioning and global regulation functions in the central control region of the IncP plasmids RK2 and R751. Microbiology 1997, 143:2167-2177.

51. Ludwiczak M, Dolowy P, Markowska A, Szarlak J, Kulinska A, Jagura-Burdzy G: Global transcriptional regulator KorC coordinates expression of three backbone modules of the broad-host-range RA3 plasmid from IncU incompatibility group. Plasmid 2013, 70:131-145.

doi:10.1186/s12866-014-0235-1

Cite this article as: Godziszewska et al: MobC of conjugative RA3 plasmid from IncU group autoregulates the expression of bicistronic mobC-nic operon and stimulates conjugative transfer. BMC Microbiology 2014 14:235.

\section{Submit your next manuscript to BioMed Central and take full advantage of:}

- Convenient online submission

- Thorough peer review

- No space constraints or color figure charges

- Immediate publication on acceptance

- Inclusion in PubMed, CAS, Scopus and Google Scholar

- Research which is freely available for redistribution

Submit your manuscript at www.biomedcentral.com/submit
Ciomed Central 